\title{
Repetitive Transcranial Magnetic Stimulation for Adolescent Major Depressive Disorder: A Focus on Neurodevelopment
}

\author{
Lindsay M. Oberman*, Megan Hynd, Dylan M. Nielson, Kenneth E. Towbin, \\ Sarah H. Lisanby and Argyris Stringaris
}

National Institute of Mental Health, Bethesda, MD, United States

Adolescent depression is a potentially lethal condition and a leading cause of disability for this age group. There is an urgent need for novel efficacious treatments since half of adolescents with depression fail to respond to current therapies and up to $70 \%$ of those who respond will relapse within 5 years. Repetitive transcranial magnetic stimulation (rTMS) has emerged as a promising treatment for major depressive disorder (MDD) in adults who do not respond to pharmacological or behavioral interventions. In contrast,

OPEN ACCESS

Edited by:

Christopher G. Davey,

The University of Melbourne, Australia

Reviewed by:

Omneya Ibrahim,

Suez Canal University, Egypt Aisha Sanober Chachar, Independent Researcher,

Karachi, Pakistan

*Correspondence:

Lindsay M. Oberman

lindsay.oberman@NIH.gov

Specialty section:

This article was submitted to Child and Adolescent Psychiatry,

a section of the journa

Frontiers in Psychiatry

Received: 16 December 2020 Accepted: 18 March 2021

Published: 13 April 2021

Citation:

Oberman LM, Hynd M, Nielson DM, Towbin KE, Lisanby SH and

Stringaris A (2021) Repetitive

Transcranial Magnetic Stimulation for Adolescent Major Depressive

Disorder: A Focus on

Neurodevelopment.

Front. Psychiatry 12:642847.

doi: 10.3389/fpsyt.2021.642847
rTMS has not demonstrated the same degree of efficacy in adolescent MDD. We argue that this is due, in part, to conceptual and methodological shortcomings in the existing literature. In our review, we first provide a neurodevelopmentally focused overview of adolescent depression. We then summarize the rTMS literature in adult and adolescent MDD focusing on both the putative mechanisms of action and neurodevelopmental factors that may influence efficacy in adolescents. We then identify limitations in the existing adolescent MDD rTMS literature and propose specific parameters and approaches that may be used to optimize efficacy in this uniquely vulnerable age group. Specifically, we suggest ways in which future studies reduce clinical and neural heterogeneity, optimize neuronavigation by drawing from functional brain imaging, apply current knowledge of rTMS parameters and neurodevelopment, and employ an experimental therapeutics platform to identify neural targets and biomarkers for response. We conclude that rTMS is worthy of further investigation. Furthermore, we suggest that following these recommendations in future studies will offer a more rigorous test of rTMS as an effective treatment for adolescent depression.

Keywords: depression, rTMS, adolescence, neurodevelopment, individualized targeting

\section{INTRODUCTION}

Adolescent depression is a leading cause of disability, yet its treatment remains unsatisfactory. Thus, there exists an urgent need for novel, neurodevelopmentally-informed, targeted therapeutics. Repetitive Transcranial magnetic stimulation (rTMS) has emerged as a promising treatment modality for adult major depressive disorder (MDD). rTMS is a non-invasive method to modulate brain network functioning through the application of pulsed magnetic fields (1). Several reviews concluded that rTMS could be a potentially safe and effective treatment for adolescent depression (2-6), however, empirical studies yield mixed results. 
Indeed, the sole large-scale $(n=103)$ randomized controlled trial (RCT), an industry-sponsored effort to extend FDA clearance for rTMS to depression in adolescents, was negative (7), that is, did not show a difference between rTMS and sham control.

In light of this trial, this review focuses on how neurodevelopment creates challenges for development of rTMS protocols in this population and provides specific recommendations to overcome these complexities. We highlight the developmental pathophysiology underlying symptoms of adolescent depression and relate these to the putative mechanisms of action of rTMS. We propose that future studies employ experimental therapeutics approaches to identify predictive biomarkers of response and to develop individualized, neurodevelopmentally informed rTMS targets.

Relevant studies were ascertained via a literature search of PubMed and Google Scholar. The search was limited to Englishlanguage peer-reviewed articles. The search terms used were: "adolescent depression" and "TMS" or "rTMS." Also, to ensure that we accurately represented the full extent of the literature, we examined previously published review articles. We excluded studies that applied single-pulse TMS, paired-pulse TMS, or other non-therapeutic TMS protocols, trials. We also excluded studies that evaluated depressive symptoms in adolescents with other primary clinical conditions [e.g., Tourette syndrome or autism spectrum disorder $(8,9)]$.

\section{A NEURODEVELOPMENTAL OVERVIEW OF ADOLESCENT DEPRESSION}

The World Health Report suggests that depression is the leading cause of disability worldwide, affecting over 264 million people $(10,11)$. The prevalence of moderate-to-severe depressive symptoms in youth between the ages of 12 and 17 is estimated to be $5.7 \%$ (12) with a cumulative prevalence of around $10 \%$ by age 16 (13). Moreover, depressed adolescents are about 30 times more likely to commit suicide compared to their non-depressed counterparts (14). Suicide is one of the leading causes of death in adolescents in the US and adolescence is also the time of peak incidence of suicidal behaviors and suicidal ideation (15). Despite the substantial individual and societal impact associated with depression in youth, treatment options are limited (16, 17). Traditional treatment methods include psychopharmacology (e.g., serotonin reuptake inhibitors) and behavioral therapy [e.g., cognitive behavioral therapy (CBT)]. The Treatment of Adolescent Depression Study (TADS), however, found that only $37 \%$ of patients experienced full remission of symptoms after 12 weeks of these first-line treatments (18). Furthermore, even with the combination of two evidence-based treatment modalities, at least a third of youths treated for depression do not respond, $20-37 \%$ only have a partial response, and $40-70 \%$ experience a relapse or recurrence (19-21). Thus, there remains a significant need for the development of new treatments.

In addition to the morbidity and mortality, there is significant financial burden of adolescent depression including: costs of health care use, productivity lost, and time off of work for caregivers. Estimates of direct costs of adolescent depression amount to $\sim \$ 2,900$ additional dollars per year. This does not consider the indirect costs of reduced/lost productivity, which in adult depression is estimated as high as $\$ 12,000$ per year (22). Given this financial burden, the cost-efficiency of treatment options is also a consideration. Pharmacotherapy, estimated at $\$ 100$ per month is the least expensive therapy, followed by psychotherapy, estimated at \$100-\$150 per session. Both pharmacotherapy and psychotherapy are significantly less expensive than rTMS for a given depressive episode. However, one industry-sponsored study of individuals who failed a single course of antidepressants, applied simulation modeling to compare costs of rTMS therapy to multiple serial medication trials and suggested that rTMS may cost less in over the course of the patients' lifetime (23).

Adolescence is not only a time when the incidence of depression increases (24), but also a period of substantial social, emotional, and biological development. These developmental changes may contribute to risk factors and mechanisms underlying adolescent depression (25). Synapses in the adolescent brain are highly dynamic; new synapses are formed and others eliminated at higher rates than seen in adults $(26,27)$. Proposed developmental models of the increased risk of psychopathology in adolescence point to a mismatch in the growth of brain networks supporting emotional reactivity and regulation. Compared to brain networks subserving emotion regulation, those pertaining to emotional reactivity develop more rapidly $(28,29)$. The prefrontal cortex (PFC) is a key node in the emotion regulation network underlying complex cognitive tasks such as inhibition, working memory, cognitive control, and attention. The PFC undergoes age-dependent functional changes well into late adolescence and early adulthood (30-32). Structural neuroimaging shows decreases in total gray matter PFC volume starting at around 11-12 years old $(33,34)$. This decrease is thought to be associated with synaptic pruning (27). In contrast, imaging metrics of myelination, axon density and white matter volume in frontal regions, show relatively linear increases across adolescence (35-39). It is postulated that an imbalance of the immature PFC and the more mature frontal subcortical systems regulating emotional reactivity might lead to a predominance of "bottom-up" emotional reactivity (40-44).

On a molecular level, these adolescent neurodevelopmental changes are thought to result from fluctuations in neurotransmitter concentration and receptor expression. Notably, fluctuations in neurotransmitter systems, such as GABA (45, 46), NMDA (47), and dopamine (48, 49) have profound impact on neural signaling in regions pertaining to emotion regulation. GABAergic and glutamatergic systems in the PFC and cingulate cortices have a direct impact on the excitability and plasticity in regions subserving emotion regulation (50). Fluctuating dopamine levels and emergence of dopamine receptor-mediated facilitation of NMDA (glutamate) receptor transmission and GABAergic interneuron excitability both have been proposed as a mechanism of increased sensitivity to rewards, novelty, or other salient stimuli (51-53). These cellular and molecular changes in adolescence lead to imbalances in excitation and inhibition, changes in cortical plasticity and 
connectivity, and less effective transferring of information between critical emotion processing brain regions $(50,54)$.

The pathophysiology that underlies adolescent depression may differ from that in adults (54). Studies in clinical samples and animal models suggest that these aberrant maturational processes contribute to adolescent depression (55). In one magnetic resonance spectroscopy (MRS) study, adolescents with depression showed decreased levels of GABA in the anterior cingulate cortex (ACC), as compared to healthy adolescents. Furthermore, this difference was specifically related to anhedonic symptoms (56). Another study found that symptom severity in both adults and adolescents with depression correlated with GABA and glutamate + glutamine (Glx) concentrations in the PFC $(57,58)$.

Neurodevelopmental changes and pathophysiology need to be considered when designing trials of novel targeted therapeutics, such as rTMS. In the next section we review the literature on rTMS. We suggest that future rTMS protocols may benefit from applying neurodevelopmentally-informed approaches of modulating aberrant brain networks and neurotransmitters to treat adolescent depression.

\section{REPETITIVE TRANSCRANIAL MAGNETIC STIMULATION IN ADULT AND ADOLESCENT MAJOR DEPRESSIVE DISORDER}

TMS is a non-invasive neuromodulation technique that is increasingly utilized in clinics and laboratories world-wide to study and treat a range of neurological and psychiatric disorders. In research, TMS can be applied in single pulses to depolarize a small population of neurons in a targeted brain region. Singlepulse TMS can be used to measure cortical excitability, study central motor conduction time, or the cortical silent period (a measure of intracortical inhibition), or map effective connectivity between the stimulated region and other brain regions (59). TMS can also be applied in pairs of pulses (i.e., paired-pulse stimulation); two pulses are presented in rapid succession to study intracortical inhibition and facilitation $(60,61)$.

During rTMS, trains of regularly repeating TMS pulses are applied at various stimulation frequencies (e.g., 1, 5, $10 \mathrm{~Hz}$ ) and patterns [e.g., Theta Burst Stimulation (TBS) (62) or Quadrapulse Stimulation (QPS) (63)]. Compared to paired-pulse or single-pulse stimulation protocols, rTMS pulses temporally summate to produce longer lasting changes in neural activity (64). Stimulation frequencies $1 \mathrm{~Hz}$ or lower are thought to produce local cortical inhibition while those $5 \mathrm{~Hz}$ or higher are thought to generate local cortical excitation $(64,65)$. There are also specific patterned forms of rTMS including intermittent theta burst stimulation (iTBS) and continuous theta burst stimulation (cTBS). iTBS and cTBS protocols lead to long-lasting facilitation and suppression of cortical excitability, respectively. Compared to $30 \mathrm{~min}$ or more for the standard $10 \mathrm{~Hz}$ rTMS procedures, a single session of cTBS and iTBS takes $\sim 40$ s and 3 min, respectively.
At a system-level, rTMS modulates excitability in targeted regions of stimulation (66-69) and exerts broader effects across networks connected to those regions (70-74). Thus, in adolescents with depression, rTMS applied to PFC could mitigate some regional prefrontal pathophysiology and aberrant functional connectivity between PFC and the limbic system. Furthermore, if successful, modulating these systems during adolescence, a critical period of PFC maturation, could potentially generate longer term clinical benefits than seen in adults. However, the degree and direction of neurophysiological effect of rTMS are influenced by the state of excitability of the targeted cortical region and the degree of functional connectivity across the targeted network $(75,76)$. In addition, as noted above, there is considerable inter-individual variability at the symptom and pathophysiological level. Thus, it is important to characterize the current brain state in terms of local cortical excitability and network connectivity in order to determine the optimal treatment protocol for a given individual. As will be described below, identifying the optimal target and protocol for a given individual remains theoretical due to the complex etiology of adolescent depression.

\section{Safety and Mechanisms of Action of rTMS in Adult and Adolescent MDD}

The safety of TMS in clinical practice and research has been evaluated through multiple meta-analyses (77-80). Safety guidelines have also been disseminated by the International Federation of Clinical Neurophysiology (81-83). Widespread application of several TMS protocols, across diverse populations and devices, show a low incidence of Adverse Events (AEs) (84). This safety record led to FDA clearance of rTMS for the treatment of adult MDD and adult obsessive-compulsive disorder (OCD) in 2008 and 2018, respectively.

Initial rTMS trials in depression were based on theory that the clinical symptoms might arise from an imbalance between PFC hypometabolism and the limbic system (85). Early studies aimed to increase excitability in regions of PFC that were thought to influence regulation of the limbic system $(66,86-88)$. Subsequent blood oxygen-level dependent (BOLD) functional magnetic resonance imaging (fMRI) studies suggest that rTMS modulates both activity and connectivity of the targeted region and related networks (74, 89-94). Of note, the cellular and molecular mechanism of action of rTMS is still under investigation (95). At a system level, the Human Connectome Project $(96,97)$ has led to development of resting state functional connectivity (RSFC) signatures that are being proposed as individualized rTMS targets (98-101). It has also been suggested that some of the changes seen on fMRI following rTMS may result from modulations in GABA and glutamatergic systems $(2,57,102-106)$.

As compared to the adult literature, the data on the safety of rTMS in adolescent depression are lacking. However, the data that exist suggest a similar safety profile in older children and adolescents as compared to adults. Allen et al. (107) conducted a systematic review of TMS safety in pediatric populations (including healthy volunteers and youth with neurological and/or psychiatric disorders) in 2017. Forty-two single-pulse and/or 
paired-pulse TMS studies $(n=1,205)$ and 26 rTMS studies ( $n$ $=360$ ) were reviewed. Adverse event rates ranged from 3.4 to $10.11 \%$ and varied based on the patient population being studied, the form of TMS being applied, and the number of sessions applied. Those with known neurological disorders or those receiving epileptogenic medications for psychiatric disorders were more at risk of adverse events. Similarly, adverse events were more common in high-frequency and/or high intensity rTMS protocols and protocols that involved a higher number of sessions (107). In 2020, Zewdie et al. (108), who run a pediatric brain stimulation clinical research program, published a report on their experience with the safety and tolerability of TMS in a cohort of 384 youth (108). The individuals in this report included healthy volunteers $(n=118)$, patients with perinatal stroke $(n=$ $101)$, patients with mild traumatic brain injury $(n=121)$, and patients with neuropsychiatric disorders $(n=37)$. They report no serious adverse events and excellent tolerability despite over a hundred patients who were at greater risk for seizure due to a neurological condition. As with previous reports, Zewdie et al. note that the most common side-effects were transient headache and neck pain. The authors conclude that standard TMS paradigms, including single pulse, paired-pulse, and rTMS, should be considered minimum risk and provide a safety and tolerability evaluation form for use in this population.

In 2015, Krishnan et al. conducted a safety review involving 35 studies $(n=322)$ focused on the use of rTMS in children and adolescents with range of conditions (109). Fifteen studies reported no adverse events or that the treatment was "welltolerated," without specifying adverse events. The most common adverse events reported were headache (11.5\% of patients) and scalp discomfort (2.5\%). A third pediatric TMS safety study was conducted by Hong and colleagues in 2015 and focused specifically on the safety and tolerability a novel rTMS paradigm, namely TBS (110). This retrospective analysis $(n=76)$ reported adverse events in $10.5 \%$ of TBS sessions including: headache, $\mathrm{arm} / \mathrm{hand} /$ other pain, numbness/tingling, and weakness (110). The rate and severity of adverse events reported in this study did not differ between those that received TBS and a comparator group of 89 youth who received single- and paired-pulse TMS. Similar rates were also reported for active and sham (placebo) TBS (110).

More serious adverse events in pediatric studies have been rare (occurring in $\sim 1-2 \%$ of participants). Two cases of syncope in children with pediatric stroke and six cases of seizures (four in adolescents with depression, one in an adolescent with migraines, and one in an adolescent with schizophrenia) have been reported (111-116). Factors that could have increased the risk of these serious adverse events include: concomitant medication use (in four of the cases), alcohol withdrawal (in one case), and clinical disorders associated with increased risk of syncope and seizure (i.e., pediatric stroke and migraine, respectively). More details about these case reports can be found in Table 1 .

The most recent International Federation of Clinical Neurophysiology TMS safety guidelines indicate that the extant pediatric literature "provide reassurance regarding the safety of these techniques" in pediatric populations (82). However, as noted above, this "reassurance" is based on far less data than in the adult literature. Furthermore, the neurodevelopmental processes ongoing in children and adolescence, compounded by the pathophysiological processes affecting those with neuropsychiatric and neurodevelopmental disorders require consideration $(117,118)$. As it relates to adolescent depression, the aforementioned neurotransmitter fluctuations and potentially aberrant functional connectivity may result in an altered neurophysiological state (as compared to the adult brain). Thus, evaluating and adjusting for the physiological state of the brain may both reduce the risk of adverse events as well as potentially increase the intended effects.

\section{Efficacy of rTMS in Adult and Adolescent MDD Efficacy of rTMS in Adult MDD}

In addition to publishing safety guidelines, the International Federation of Clinical Neurophysiology has also published a series of evidence-based guidelines on the therapeutic use of rTMS $(119,120)$. To develop these guidelines, experts in the field evaluated the level of evidence of rTMS efficacy for a number of indications. Consistent with the FDA label and based on a number of large-scale clinical trials (121-123), high-frequency $(10 \mathrm{~Hz})$ rTMS to the left dorsolateral prefrontal cortex (DLPFC) achieved a "Level A" (definite efficacy) for adult MDD. Since its initial FDA clearance, in 2008, multiple TMS devices and protocols have also received clearance for adult MDD. Consensus guidelines have also been established by the National Network of Depression Centers and the American Psychiatric Association Council on Research (124). Multiple meta-analyses of thousands of individuals have concluded that rTMS is safe, tolerable, and leads to a reduction in depressive symptoms in otherwise treatment resistant adult patients with MDD. Although the safety and tolerability of rTMS is consistent across trials, effect sizes vary greatly based on a number of factors including: rTMS parameters (e.g., intensity, location, and stimulation protocol) and interindividual factors such as brain size, shape, and neurophysiological state.

Intensity of stimulation is typically set in relation to the individual's motor threshold (MT). The MT is the stimulator output that is required to produce a contraction of the thumb or fingers half the time when applied to the primary motor cortex "hotspot." MT is used as a proxy of the intensity of stimulation necessary to activate other regions of cortex. Intensity of rTMS typically ranges from 80 to $120 \%$ of MT. At these intensity levels, current models indicate that standard coils induce an electrical field that can reach $2-3 \mathrm{~cm}$ from the scalp (125). Consistent with the FDA label for adult MDD, the DLPFC target is often approximated from measurements on the scalp (126). However, some studies have also used structural or functional MRI combined with a frameless stereotaxic neuronavigation system to target specific regions of interest $(65,127)$. Targeting rTMS based on fMRI and/or diffusion tensor imaging (DTI) mapping of an individual's brain network $(128,129)$ tends to result in larger effect sizes as compared to scalp-based approaches $(127,130,131)$. Typical stimulation frequencies vary from 1 to $20 \mathrm{~Hz}$. The most common frequency used for adult MDD 
TABLE 1 | Case reports of TMS induced seizures in adolescents.

\begin{tabular}{|c|c|c|c|c|c|c|c|}
\hline Publication & Patient status & Age, gender & TMS protocol & Intensity & Location & $\begin{array}{l}\text { Stimulator model/ } \\
\text { Coil type }\end{array}$ & Seizure description \\
\hline $\begin{array}{l}\text { Hu et al. (111) } \\
\text { Journal of International } \\
\text { Medical Research }\end{array}$ & MDD & $15(F)$ & $10 \mathrm{~Hz}$ rTMS & $100 \% \mathrm{RMT}$ & L- Prefrontal Lobe & $\begin{array}{l}\text { Magstim Figure } \\
8 \text { Coil }\end{array}$ & $\begin{array}{l}\text { Generalized tonic-clonic } \\
\text { seizure, started within } \\
\text { minutes of } 1 \text { st treatment }\end{array}$ \\
\hline $\begin{array}{l}\text { Chiramberro et al. } \\
(112) \\
\text { Brain Stimulation }\end{array}$ & MDD & $16(F)$ & $10 \mathrm{~Hz}$ rTMS & Not reported & L-DLPFC & $\begin{array}{l}\text { Magstim Figure } \\
8 \text { Coil }\end{array}$ & $\begin{array}{l}\text { Generalized tonic-clonic } \\
\text { seizure induced } 20 \text { min } \\
\text { into } 40 \text { trains on the } \\
\text { 12th day of stimulation }\end{array}$ \\
\hline $\begin{array}{l}\text { Cullen et al. (113) } \\
\text { Journal of Child and } \\
\text { Adolescent } \\
\text { Psychopharmacology }\end{array}$ & MDD & $17(F)$ & 18 Hz Deep TMS & $120 \% \mathrm{RMT}$ & L- Motor cortex & H1 Coil & $\begin{array}{l}\text { Generalized tonic-clonic } \\
\text { seizure induced on the } \\
\text { 48th train of the 8th day } \\
\text { of treatment }\end{array}$ \\
\hline $\begin{array}{l}\text { Wang et al. } \\
(114) \\
\text { Brain Stimulation }\end{array}$ & Migraine & $16(F)$ & $10 \mathrm{~Hz}$ rTMS & $110 \% \mathrm{RMT}$ & L- Motor cortex & $\begin{array}{l}\text { Magstim Rapid Figure } \\
\text { of } 8 \text { Coil }\end{array}$ & $\begin{array}{l}\text { Generlized tonic-clonic } \\
\text { seizure induced } 10 \\
\text { seconds into the } 3 \text { rd } \\
\text { train of the 1st session }\end{array}$ \\
\hline $\begin{array}{l}\text { Purushotham et al. } \\
\text { (115) } \\
\text { Brain Stimulation }\end{array}$ & Schizophrenia & $15(F)$ & iTBS & $80 \%$ AMT & L-Motor cortex & $\begin{array}{l}\text { Magstim Rapid Figure } \\
\text { of } 8 \text { Coil }\end{array}$ & $\begin{array}{l}\text { Generalized tonic-clonic } \\
\text { seizure induced } 30 \\
\text { seconds into the } 1 \text { st } \\
\text { session }\end{array}$ \\
\hline $\begin{array}{l}\text { Kallel and Brunelin } \\
\text { (116) } \\
\text { Journal of ECT }\end{array}$ & MDD & $18(F)$ & $20 \mathrm{~Hz}$ rTMS & $110 \% \mathrm{RMT}$ & L-DLPFC & $\begin{array}{l}\text { MagPro X30 Figure of } \\
8 \text { Coil }\end{array}$ & $\begin{array}{l}\text { Generlized tonic-clonic } \\
\text { seizure induced on the } \\
26 \text { th train of the } 3 \text { rd } \\
\text { session on the } 2 \text { nd day } \\
\text { of stimulation }\end{array}$ \\
\hline
\end{tabular}

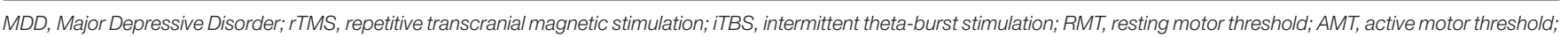
$L-D L P F C$, left dorsolateral prefrontal cortex.

is $10-\mathrm{Hz}$. rTMS applied to the DLPFC at $10 \mathrm{~Hz}$ frequency per the FDA label is associated with an average of $30 \%$ response rate, compared with $10.4 \%$ with sham (placebo) rTMS, with an effect size of $0.55(132-134)$ and a pooled odds ratio of response or remission of 3.3 (132). Furthermore, according to a recent meta-analysis, $66.5 \%$ of individuals who respond to the initial rTMS course have sustained response after 3 months, with responder rates decreasing to $52.9 \%$ after 6 months, and $46.3 \%$ of individuals still maintaining response 1 year after the initial treatment course (135).

Several approaches have been taken to address relapse and recurrence after initial rTMS response. These include various maintenance rTMS schedules as well as additional courses of rTMS treatments during periods of relapse. Though a number of studies have explored various maintenance rTMS schedules [see (136)], protocols vary across studies and clinics. Proposed maintenance regimen involve an initial tapering of sessions over the course of 3-4 weeks from five sessions per week down to one session every week and eventually one session every 2 or 3 weeks for many months to several years depending on the individual [see (137)]. Though this regimen may provide optimal protection against relapse, there may be alternative options for maintenance that are less burdensome including introduction and/or modifications of antidepressant medication or psychotherapy.

The effects of rTMS are not simply a matter of the stimulation parameters, but also how the stimulation is received and processed in the brain. The specific degree and location of stimulation of the targeted brain region depends upon the individually unique structural and functional architecture of each individual's brain. Given the putative mechanism of action of rTMS is the modulation of functional networks and the known interindividual structural and functional heterogeneity of these networks, the efficacy of rTMS depends upon the accurate targeting of the network of interest in that individual. Furthermore, although high-frequency and iTBS protocols typically lead to increased excitability of the targeted region, recent studies report considerable inter-individual and intraindividual (state-dependent) variability in cortical response, especially outside of the primary motor cortex $(138,139)$. Thus, efficacy can be optimized through careful characterization of both the functional network architecture and neurophysiological state.

\section{Efficacy of rTMS in Adolescent MDD}

Most of what is known about the effects of rTMS on the brain are based on adult studies. Less than 1,000 children and adolescents are represented in the published rTMS literature. Without FDA clearance, commercial marketing of rTMS for any pediatric indications is prohibited. While adolescents and children may be studied under an approved research protocol, any other use of TMS in individuals under age 21 is "off-label" in the United States.

The first published review on rTMS in adolescent depression (6) was based on two case series $(140,141)$ and one open-label 
trial (142) ( $n=14)$. With so few data, no meaningful conclusions could be made about efficacy. The authors suggested that the optimal stimulation parameters for adolescents might differ from those in adults. A subsequent review (3) included another openlabel trial (143), a case report of an induced seizure in an adolescent (111), an open-label trial where depressive symptoms were evaluated in adolescents with Tourette syndrome (8), and a secondary analysis on the previous open-label trial. Despite the expanded number of publications, the rTMS literature was comprised of only 22 (inclusive of the one seizure report) adolescents with a primary diagnosis of MDD. A third review, a decade after the first (4), added three additional case reports $(112,113,144)$, a case report of an individual with autism spectrum disorder and co-morbid depression who received rTMS for depressive symptoms (9), a case series (106), an open-label study (58), and three secondary analyses on previous datasets (145-147). By 2017, despite more publications, $<50$ adolescents with primary MDD had received rTMS and no studies included a placebo control group. The next review (2), in 2019, included two additional open-label trials $(148,149)$, more than doubling the previous total number of depressed adolescents treated with rTMS. This review focused on the effects of rTMS on GABAergic and glutamatergic neurotransmission and concluded by calling for larger, neurodevelopmentally-informed studies. The most recent review (5), included one additional case series (150), one new retrospective analysis of clinical data (151), and two more secondary analyses of existing datasets $(152,153)(n=\sim 150)$. The authors (5) conclude by highlighting flawed study designs, calling for sham-controlled RCTs to properly assess the efficacy of rTMS.

Since the publication of the most recent review article, there is now one sham-controlled RCT of rTMS in adolescents with MDD (7) and two additional retrospective analyses of clinical data $(154,155)$. With the addition of these new data, the adolescent depression rTMS literature now encompasses 20 publications (Table 2) representing 12 unique datasets ( $n$ $=\sim 280$ adolescent participants with MDD). Despite the calls for neurodevelopmentally-informed trials, the parameters have mimicked those in the adult studies. Intensity of stimulation has ranged from 80 to $120 \%$ of motor threshold. Protocols have largely followed the FDA clearance (for adults) applying $10 \mathrm{~Hz}$ unilaterally to the left DLPFC. Two studies took a different path. One (151) compared $1 \mathrm{~Hz}$, unilateral right DLPFC stimulation with a bilateral (left followed by right) stimulation and another applied bilateral TBS stimulation (148). As for sample characteristics, studies have taken a conservative approach, enrolling relatively older adolescents (average age of 17.15), small sample sizes ( $7 / 12$ datasets with sample sizes $n \leq 10)$, and openlabel designs or active stimulation case reports/retrospective analyses of clinical data (11/12 datasets). The sole exception is the large-scale RCT that enrolled 103 adolescents $\sim 18 \%$ of whom were age 12-14 years old (7).

Acknowledging the limitations of the method, open-label trials and case series/retrospective analyses of clinical data were generally positive, with large effect sizes open-label trials: Hedges's $\mathrm{g}_{\mathrm{av}}=2.39$ and Glass's $\Delta_{\mathrm{pre}}=3.01$; case reports/retrospective analyses: Hedges's $\mathrm{g}_{\mathrm{av}}=2.06$ and Glass's
$\Delta_{\text {pre }}=3.48$, [see (158) for explanation of effect size measures]. Average reduction in depressive symptoms for the open-label trials and case series/retrospective analyses equals 40 and 51\%, respectively (with study averages ranging from 23 to $71 \%$ ). Open-label trials and case series/retrospective analyses inherently inflate effect sizes and are influenced by regression to the mean, investigator biases, and confounding the effect of the active treatment with placebo effects (159). The only large-scale RCT of rTMS in adolescent depression yielded an effect size near zero (Hedges's $g=0.10$ ). The active and sham rTMS groups showed comparable response $(41.7 \%$ for the active and $36.4 \%$ for the sham group) and remission rates (29.2\% for the active and $29.0 \%$ for the sham group) (7).

Sham TMS coils have been used in rTMS trials as an analog to pill placebo in drug trials. Sham coils do not apply a magnetic field but mimic the auditory, visual, and (in some cases) sensory experience of rTMS (160). The largest placebo effects have been seen in trials employing "physical placebo interventions" (e.g., a sham TMS coil) with subjective patient-related outcomes (e.g., self or parent reports or clinician administered interviews) (161). A meta-analysis of 61 studies using rTMS sham-controls for adult depression found a placebo effect size of 0.8 with the magnitude of the effect positively correlated with year of publication (162). Increasing placebo response over time may be an outgrowth of public/media attention, technologically more sophisticated devices (such as neuronavigation), enrollment of patients who are not "treatment resistant" $(163,164)$, and improved disguising of sham coils (165). The RCT in adolescents resulted in a sham effect size of 1.15 and a response rate of $36.4 \%$ (not significantly lower than the active rTMS effect size of 1.27 and response rate of $41.7 \%)$ (7). This higher placebo response rate in adolescents is also present in drug trials for adolescent depression (166) where placebo responses rates range from 24 to $60 \%$ (167-174).

To determine the efficacy of rTMS for adolescent depression, future RCTs will have to address the higher placebo effect rate. Aside from the conventional "active vs. sham" design alternative approaches such as inclusion of a placebo run-in, giving an active comparator, or applying statistical analysis techniques such as Growth Mixture Modeling (GMM) to capture unobserved subject heterogeneity in trajectories may be considered. The last approach requires dense collection of outcome measures over the course of rTMS and during followup. GMM was used to analyze data from an antidepressant trial; the placebo response trajectory deviated from the two active drug response trajectories (175). This technique has also been used to evaluate whether biomarkers, such as quantitative electroencephalography (qEEG), can predict antidepressant response (176).

Beyond the placebo response rate, other features in the study design for the RCT may have contributed to the failed outcome, including: the choice of the " $5-\mathrm{cm}$ rule" targeting strategy, broad inclusion criteria, within group variability in response, and insufficient sample size. The rTMS protocol, targeting strategy, frequency of stimulation, and dosage influence effect size in rTMS trials. Optimizing these factors in a neurodevelopmentally informed way could therefore increase effect sizes. 
TABLE 2 | Previous literature on TMS for adolescent depression.

\begin{tabular}{|c|c|c|c|c|c|c|c|c|c|c|}
\hline Publication & $n$ & $\begin{array}{l}\text { Age in years } \\
\text { (Mean, } \\
\text { Range) }\end{array}$ & Gender & Protocol & $\begin{array}{l}\text { Number of } \\
\text { sessions, } \\
\text { Frequency of } \\
\text { sessions }\end{array}$ & $\begin{array}{l}\text { Depression } \\
\text { outcome } \\
\text { measure }\end{array}$ & $\begin{array}{l}\text { Subject } \\
\text { medications }\end{array}$ & $\begin{array}{l}\text { Estimated } \\
\text { effect size }\end{array}$ & $\begin{array}{l}\text { Reported effects for } \\
\text { depression } \\
\text { outcome measure }\end{array}$ & $\begin{array}{l}\text { Side- } \\
\text { effects/Adverse } \\
\text { events }\end{array}$ \\
\hline $\begin{array}{l}\text { Walter et al. (141) } \\
\text { Journal of Child and } \\
\text { Adolescent } \\
\text { Psychopharmacology }\end{array}$ & $n=3$ & $\begin{array}{l}\text { Ages: } 16,17 \text {, } \\
\text { and } 17\end{array}$ & 3 males & $\begin{array}{l}10 \mathrm{~Hz} \text { rTMS, } \\
\text { 90-110\% RMT, over } \\
\text { LDLPFC }\end{array}$ & $\begin{array}{l}10 \text { treatment } \\
\text { sessions over } 2 \\
\text { weeks }\end{array}$ & HAM-D \& BDI & None & $\begin{array}{l}\text { Hedges's gav }= \\
1.53, \text { Glass's } \Delta_{\text {pre }} \\
=3.37\end{array}$ & $\begin{array}{l}\text { Improvement of } \\
\text { HAM-D from } 28 \\
\text { (baseline) to } 8 \text { (week } \\
\text { 4) for one participant } \\
\text { Improvement of } \\
\text { HAM-D from } 34 \\
\text { (baseline) to } 12 \text { (week } \\
\text { 4) for one participant } \\
\text { No improvement for } \\
\text { one participant }\end{array}$ & $\begin{array}{l}\text { Adverse effects in } \\
\text { only one } \\
\text { patient-tension } \\
\text { headache in two } \\
\text { sessions }\end{array}$ \\
\hline $\begin{array}{l}\text { Loo et al. (140) } \\
\text { Australasian Psychiatry }\end{array}$ & $n=2$ & Ages: 16, 16 & Both female & $\begin{array}{l}10 \mathrm{~Hz} \text { rTMS at } 110 \% \\
\text { RMT; } 40 \text { trains of } 5 \\
\text { second duration, } 25 \\
\text { second ITI }\end{array}$ & $\begin{array}{l}29-36 \text { treatment } \\
\text { sessions over } \\
6-11 \text { weeks }\end{array}$ & $\begin{array}{l}\text { MADRS, CGIS, } \\
\text { BDI, Centre for } \\
\text { Epidemiological } \\
\text { Studies- } \\
\text { Depression-Child } \\
\text { Scale }\end{array}$ & $\begin{array}{l}n=1: \text { "psychotropic } \\
\text { medication," } n=1 \text { : } \\
\text { venlafaxine and } \\
\text { methylphenidate }\end{array}$ & $\mathrm{n} / \mathrm{a}$ & $\begin{array}{l}\text { "Both subjects } \\
\text { improved to a clinically } \\
\text { significant degree with } \\
\text { rTMS treatment }\end{array}$ & No adverse effects \\
\hline $\begin{array}{l}\text { Bloch et al. (142) } \\
\text { The Journal of ECT } \\
\text { *Mayer et al. (156) }\end{array}$ & $n=9$ & $\begin{array}{l}M=17.3 \\
\text { Range } \\
=16-18\end{array}$ & $\begin{array}{l}2 \text { males, } 7 \\
\text { females }\end{array}$ & $\begin{array}{l}10 \mathrm{~Hz} \text { rTMS at } 80 \% \\
\text { RMT over LDLPFC } \\
\text { ( } 5 \mathrm{~cm} \text { targeted); } 20 \\
\text { trains, } 2 \mathrm{~s} \text { per train }\end{array}$ & $\begin{array}{l}20 \text { treatment } \\
\text { sessions over } 2 \\
\text { weeks }\end{array}$ & $\begin{array}{l}\text { CDRS, Screen for } \\
\text { Child } \\
\text { Anxiety-Related } \\
\text { Disorders, Suicidal } \\
\text { Ideation } \\
\text { Questionnaire } \\
\text { CGIS,Cambridge } \\
\text { Neuropsychological } \\
\text { Test } \\
\text { Automated Battery }\end{array}$ & Not reported & $\begin{array}{l}\text { Hedges's gav }= \\
1.50, \text { Glass's } \Delta_{\text {pre }} \\
=2.63\end{array}$ & Response rate of $33 \%$ & $\begin{array}{l}\text { No adverse effects } \\
\text { reported }\end{array}$ \\
\hline $\begin{array}{l}\text { Wall et al. (143) } \\
\text { The Journal of Clinical } \\
\text { Psychiatry } \\
{ }^{*} \text { Croarkin et al. (147) } \\
{ }^{*} \text { Wall et al. (146) } \\
{ }^{*} \text { Croarkin et al. (153) } \\
\text { *Somnez et al. (157) }\end{array}$ & $n=7$ & $\begin{array}{l}M=16.5 \\
\text { Range =14.6- } \\
17.8\end{array}$ & $\begin{array}{l}1 \text { male, } 6 \\
\text { females }\end{array}$ & $\begin{array}{l}10 \mathrm{~Hz} \text { rTMS at } 120 \% \\
\text { RMT over LDLPFC } \\
(5 \mathrm{~cm} \text { targeted); train } \\
\text { duration of } 4 \mathrm{~s}, 26 \mathrm{~s} \\
\text { ITI, total } 3,000 \text { pulses }\end{array}$ & $\begin{array}{l}30 \text { treatment } \\
\text { sessions over } \\
6-8 \text { weeks }\end{array}$ & $\begin{array}{l}\text { CDRS-R, } \\
\text { QIDS-A17, CGI-S, } \\
\text { Suicide Severity } \\
\text { Scale }\end{array}$ & Not reported & $\begin{array}{l}\text { Hedges's gav }= \\
4.51, \text { Glass's } \Delta_{\text {pre }} \\
=5.05\end{array}$ & $\begin{array}{l}\text { CDRS-R scores } \\
\text { improved from } \\
\text { treatment } 10 \text { (mean = } \\
50.9, \mathrm{SD}=12, P< \\
0.02 \text { ) to treatment } 30 \\
\text { (mean }=32.6, \mathrm{SD}= \\
7.3, P<0.0001 \text { ), and } \\
\text { at } 6 \text {-month follow-up } \\
\text { (mean }=32.7, \mathrm{SD}= \\
3.8, P<0.0001 \text { ) }\end{array}$ & $\begin{array}{l}\text { Scalp discomfort in } \\
3 \text { out of } 8 \\
\text { participants }\end{array}$ \\
\hline $\begin{array}{l}\text { Yang et al. (106) } \\
\text { The Journal of ECT }\end{array}$ & $n=6$ & $\begin{array}{l}M=18.7 \\
\text { Range }= \\
15-21\end{array}$ & $\begin{array}{l}2 \text { males, } 4 \\
\text { females }\end{array}$ & $\begin{array}{l}10 \mathrm{~Hz} \text { rTMS at } 120 \% \\
\text { RMT over LDLPFC } \\
\text { (structural-MRI } \\
\text { targeted); } 4 \mathrm{~s} \text { trains, } \\
\text { ITI } 26 \mathrm{~s}, 75 \text { trains, } \\
\text { 3,000 pulses }\end{array}$ & $\begin{array}{l}15 \text { treatment } \\
\text { sessions over } 3 \\
\text { weeks }\end{array}$ & HAM-D, BDI & Not reported & $\begin{array}{l}\text { Hedges's gav }= \\
2.63, \text { Glass's } \Delta_{\text {pre }} \\
=3.18\end{array}$ & $\begin{array}{l}\text { Response rate of } 66 \% \\
\text { Responders had an } \\
11 \% \text { increase in } \\
\text { glutamate levels } \\
\text { from baseline }\end{array}$ & $\begin{array}{l}\text { No adverse events } \\
\text { reported }\end{array}$ \\
\hline
\end{tabular}


TABLE 2 | Continued

\begin{tabular}{|c|c|c|c|c|c|c|c|c|c|c|}
\hline Publication & $n$ & $\begin{array}{l}\text { Age in years } \\
\text { (Mean, } \\
\text { Range) }\end{array}$ & Gender & Protocol & $\begin{array}{l}\text { Number of } \\
\text { sessions, } \\
\text { Frequency of } \\
\text { sessions }\end{array}$ & $\begin{array}{l}\text { Depression } \\
\text { outcome } \\
\text { measure }\end{array}$ & $\begin{array}{l}\text { Subject } \\
\text { medications }\end{array}$ & $\begin{array}{l}\text { Estimated } \\
\text { effect size }\end{array}$ & $\begin{array}{l}\text { Reported effects for } \\
\text { depression } \\
\text { outcome measure }\end{array}$ & $\begin{array}{l}\text { Side- } \\
\text { effects/Adverse } \\
\text { events }\end{array}$ \\
\hline $\begin{array}{l}\text { Segev et al. (144) } \\
\text { The Journal of ECT }\end{array}$ & $n=1$ & 17 & 1 male & $\begin{array}{l}10 \mathrm{~Hz} \text { rTMS, } 100 \% \\
\text { RMT over LDLPFC } \\
\text { ( } 5 \mathrm{~cm} \text { targeted), } 42 \\
\text { trains of } 4 \mathrm{~s} \text { with an } \\
\text { ITI of } 30 \mathrm{~s}, 1,680 \\
\text { pulses per treatment }\end{array}$ & $\begin{array}{l}20 \text { treatment } \\
\text { sessions over } 4 \\
\text { weeks }\end{array}$ & $\begin{array}{l}\text { BDI-II, SIQ, } \\
\text { Childhood Anxiety } \\
\text { Related Disorder } \\
\text { Questionnaire }\end{array}$ & Not reported & $\mathrm{n} / \mathrm{a}$ & $\begin{array}{l}\text { "...significant clinical } \\
\text { improvement was } \\
\text { demonstrated in } \\
\text { anxiety symptoms and } \\
\text { not in clinical } \\
\text { measures of } \\
\text { depression" }\end{array}$ & $\begin{array}{l}\text { Headache, scalp } \\
\text { pain, and scalp } \\
\text { burning }\end{array}$ \\
\hline $\begin{array}{l}\text { Croarkin et al. (58) } \\
\text { Psychiatry Research: } \\
\text { Neuroimaging } \\
{ }^{*} \text { Wall et al. (145) } \\
{ }^{*} \text { Croarkin et al. (153) } \\
{ }^{*} \text { Sonmez et al. (152) }\end{array}$ & $n=10$ & $\begin{array}{l}M=15.4 \\
\text { Range } \\
=13.9-17.4\end{array}$ & $\begin{array}{l}6 \text { males, } 4 \\
\text { females }\end{array}$ & $\begin{array}{l}10 \mathrm{~Hz} \text { rTMS at } 120 \% \\
\text { RMT over LDLPFC } \\
\text { (structural-MRI } \\
\text { targeted); train } \\
\text { duration of } 4 \mathrm{~s}, 26 \mathrm{~s} \\
\text { ITI, total } 3,000 \text { pulses }\end{array}$ & $\begin{array}{l}30 \text { treatment } \\
\text { sessions over } \\
6-8 \text { weeks }\end{array}$ & $\begin{array}{l}\text { CDRS-R, } \\
\text { QIDS-A17-SR, } \\
\text { CGI-S }\end{array}$ & Not reported & $\begin{array}{l}\text { Hedges's gav }= \\
1.89, \text { Glass's } \Delta_{\text {pre }} \\
=2.57\end{array}$ & $\begin{array}{l}\text { CDRS-R total score at } \\
\text { baseline was } 62.9 \text { (SD } \\
=8.2) \text {, total score at } \\
\text { posttreatment was } \\
41.8 \text { (SD = 13.2), total } \\
\text { score at 6-month } \\
\text { follow up was } 34.2 \\
\text { (SD = 15.3) } \\
\text { Also reported, } \\
\text { "...throughout the } \\
6 \text {-month follow-up } \\
\text { period, we estimated } \\
\text { that a 1-scale unit } \\
\text { increase (or decrease) } \\
\text { in the CDRS-R total } \\
\text { score (depression } \\
\text { severity) was related } \\
\text { to a mean decrease } \\
\text { (or increase) in each } \\
\text { Gin/Glu ratio" }\end{array}$ & $\begin{array}{l}\text { Scalp discomfort, } \\
\text { headaches, } \\
\text { dizziness, neck } \\
\text { stiffness, eye } \\
\text { twitching, nausea, } \\
\text { musculoskeletal } \\
\text { discomfort }\end{array}$ \\
\hline $\begin{array}{l}\text { MacMaster et al. (149) } \\
\text { Frontiers in Psychiatry }\end{array}$ & $n=32$ & $\begin{array}{l}M=17.57 \\
\text { Range } \\
=13-21\end{array}$ & $\begin{array}{l}17 \text { males, } \\
15 \text { females }\end{array}$ & $\begin{array}{l}10 \mathrm{~Hz} \text { rTMS at } 120 \% \\
\text { over LDLPFC } \\
\text { (structural-MRI } \\
\text { targeted); } 4 \mathrm{~s} \text { trains, } \\
\text { ITI } 26 \mathrm{~s}, 75 \text { trains, } \\
3,000 \text { pulses }\end{array}$ & $\begin{array}{l}15 \text { treatment } \\
\text { sessions over } 3 \\
\text { weeks }\end{array}$ & HAM-D & Not reported & $\begin{array}{l}\text { Hedges's gav }= \\
1.82, \text { Glass's } \\
\Delta \text { pre }=1.71\end{array}$ & $\begin{array}{l}\text { Response rate of } 56 \% \\
\text { Remission rate of } 44 \%\end{array}$ & $\begin{array}{l}\text { Limiting headaches } \\
\text { and mild neck pain }\end{array}$ \\
\hline $\begin{array}{l}\text { Zhang et al., pooled } \\
\text { analysis } \\
\text { *Zhang et al. (150) } \\
\text { Brain Stimulation } \\
{ }^{\star} \text { Zhang et al. (155) } \\
\text { Journal of Affective } \\
\text { Disorders } \\
\text { *Zhang et al. (154) } \\
\text { Journal of ECT }\end{array}$ & $\begin{array}{l}n=70 \\
2 \text { weeks } \\
n=23 \\
4 \text { weeks }\end{array}$ & $\begin{array}{l}M=14.86 \\
\text { Range } \\
=10-17\end{array}$ & $\begin{array}{l}26 \text { males, } \\
44 \text { females }\end{array}$ & $\begin{array}{l}10 \mathrm{~Hz} \text { rTMS at } 120 \% \\
\text { MT over LDLPFC } \\
(5 \mathrm{~cm} \text { targeted); } 80 \\
\text { trains, } 30 \text { pulses per } \\
\text { train, } 12 \mathrm{~s} \text { ITI, } 2,400 \\
\text { pulses or } 1 \mathrm{~Hz} \text { rTMS } \\
\text { at } 120 \% \mathrm{MT} \text { over } \\
\text { RDLPFC ( } 5 \mathrm{~cm} \\
\text { targeted); } 2 \text { trains, } \\
700 \text { pulses per train, } \\
1 \mathrm{~s} \text { ITI, } 1,400 \text { pulses }\end{array}$ & $\begin{array}{l}20 \text { treatment } \\
\text { sessions over } 4 \\
\text { weeks }\end{array}$ & HAM-D \& HAMA & $\begin{array}{l}\text { Sertraline, } \\
\text { venlafaxine, } \\
\text { duloxetine, } \\
\text { mirtazapine } n=1 \text { : } \\
\text { agomelatine, } \\
\text { bupropion, deanxit. } \\
\text { and clomipramine }\end{array}$ & $\begin{array}{l}\text { Hedges's gav } 2 \\
\text { weeks }=1.65, \\
\text { Glass's } \Delta_{\text {pre }} 2 \\
\text { weeks }=1.40 \\
\text { Hedges's gav } 4 \\
\text { weeks }=2.85, \\
\text { Glass's } \Delta_{\text {pre }} 4 \\
\text { weeks }=1.98\end{array}$ & $\begin{array}{l}\text { 2-week response rate } \\
\text { of } 50 \% \\
2 \text {-week remission rate } \\
\text { of } 54.3 \% \\
4 \text {-week response rate } \\
\text { of } 100 \% \\
4 \text {-week remission rate } \\
\text { of } 91.3 \%\end{array}$ & $\begin{array}{l}\text { No serious adverse } \\
\text { events reported. } \\
\text { Limited headaches } \\
\text { or musculoskeletal } \\
\text { discomfort }\end{array}$ \\
\hline
\end{tabular}


TABLE 2 | Continued

\begin{tabular}{|c|c|c|c|c|c|c|c|c|c|c|}
\hline Publication & $n$ & $\begin{array}{l}\text { Age in years } \\
\text { (Mean, } \\
\text { Range) }\end{array}$ & Gender & Protocol & $\begin{array}{l}\text { Number of } \\
\text { sessions, } \\
\text { Frequency of } \\
\text { sessions }\end{array}$ & $\begin{array}{l}\text { Depression } \\
\text { outcome } \\
\text { measure }\end{array}$ & $\begin{array}{l}\text { Subject } \\
\text { medications }\end{array}$ & $\begin{array}{l}\text { Estimated } \\
\text { effect size }\end{array}$ & $\begin{array}{l}\text { Reported effects for } \\
\text { depression } \\
\text { outcome measure }\end{array}$ & $\begin{array}{l}\text { Side- } \\
\text { effects/Adverse } \\
\text { events }\end{array}$ \\
\hline $\begin{array}{l}\text { Rosenich et al. (151) } \\
\text { Early Intervention in } \\
\text { Psychiatry }\end{array}$ & $n=15$ & $\begin{array}{l}M=20.69 \\
\text { Range } \\
=17-25\end{array}$ & $\begin{array}{l}7 \text { males, } 8 \\
\text { females }\end{array}$ & $\begin{array}{l}\text { Unilateral treatment } \\
=\text { continuous } 1 \mathrm{~Hz} \\
\text { rTMS over RDLPFC } \\
\text { for } 15 \mathrm{~min}(n=2) \text { or } \\
30 \text { min }(n=9) \text {; } \\
\text { Bilateral treatment ( } n \\
=4)=\text { intermittent } \\
10 \mathrm{~Hz} \text { rTMS } 5 \mathrm{~s} \\
\text { intervals, } 25 \mathrm{~s} \text { ITI for } \\
1,500 \text { pulses over } \\
\text { LDLPFC and } \\
\text { followed by } 15 \text { min of } \\
1 \mathrm{~Hz} \text { unilateral } \\
\text { treatment for } 900 \\
\text { pulses over RDLPFC } \\
\text { (all } 6 \mathrm{~cm} \text { targeted). All } \\
\text { stimulation at } 110 \% \\
\text { RMT }\end{array}$ & $\begin{array}{l}18 \text { treatment } \\
\text { sessions over } 6 \\
\text { weeks }\end{array}$ & $\begin{array}{l}\text { HAM-D, MADRS, } \\
\text { and Zung Self } \\
\text { Rating Depression } \\
\text { Scale }\end{array}$ & Not reported & $\begin{array}{l}\text { Hedges's gav }= \\
1.24, \text { Glass's } \Delta_{\text {pre }} \\
=1.41\end{array}$ & $\begin{array}{l}\text { Partial response rate } \\
\text { of } 86.7 \% \\
\text { Response rate of } 40 \% \\
\text { Remission rate of } 13 \%\end{array}$ & $\begin{array}{l}\text { No serious adverse } \\
\text { events, only mild } \\
\text { headache, fatigue, } \\
\text { and localized } \\
\text { discomfort }\end{array}$ \\
\hline $\begin{array}{l}\text { Dhami et al. (148) } \\
\text { Journal of Affective } \\
\text { Disorders }\end{array}$ & $n=20$ & $\begin{array}{l}M=20.9 \\
\text { Range = } \\
16-24\end{array}$ & $\begin{array}{l}10 \text { males, } \\
10 \text { females }\end{array}$ & $\begin{array}{l}\text { Bilateral theta burst } \\
\text { stimulation: iTBS on } \\
\text { LDLPFC and cTBS } \\
\text { on RDLPFC at } 80 \% \\
\text { RMT (structural-MRI } \\
\text { targeted) }\end{array}$ & $\begin{array}{l}10 \text { treatment } \\
\text { sessions over } 2 \\
\text { weeks }\end{array}$ & $\begin{array}{l}\text { HRSD-17, BDI-II, } \\
\text { Q-LES-Q, } \\
\text { CDRS-R }\end{array}$ & Not reported & $\begin{array}{l}\text { Hedges's gav }= \\
2.21, \text { Glass's } \\
\Delta \text { pre }=3.07\end{array}$ & $\begin{array}{l}\text { Response rate of } 20 \% \\
\text { Remission rate of } 10 \%\end{array}$ & $\begin{array}{l}\text { Headache, scalp } \\
\text { pain, chest } \\
\text { tightness, anxiety, } \\
\text { nausea, } \\
\text { gastrointestinal } \\
\text { symptoms, } \\
\text { nasopharyngitis, } \\
\text { restlessness, } \\
\text { general discomfort }\end{array}$ \\
\hline $\begin{array}{l}\text { Croarkin et al. (7) } \\
\text { Neuropsychopharmacology } \\
\text { Active arm }\end{array}$ & $n=48$ & $\begin{array}{l}M=17.6 \\
\text { Range }= \\
12-21\end{array}$ & $\begin{array}{l}18 \text { males, } \\
30 \text { females }\end{array}$ & $\begin{array}{l}10 \mathrm{~Hz} \text { rTMS at } 120 \% \\
\text { over LDLPFC ( } 5 \mathrm{~cm} \\
\text { targeted); } 4 \text { strains, } \\
26 \mathrm{~s} \mathrm{ITI,} 75 \text { trains, } \\
\text { total 3,000 pulses }\end{array}$ & $\begin{array}{l}30 \text { treatment } \\
\text { sessions over } 6 \\
\text { weeks }\end{array}$ & $\begin{array}{l}\text { HAM-D, MADRS, } \\
\text { CRS-R, } \\
\text { QIDS-A-SR, } \\
\text { CGI-S }\end{array}$ & $\begin{array}{l}\text { zaleplon, zolpidem, } \\
\text { zopiclone, or } \\
\text { lorazepam }\end{array}$ & $\begin{array}{l}\text { Hedges's gav }= \\
1.27, \text { Glass's } \Delta_{\text {pre }} \\
=1.86\end{array}$ & $\begin{array}{l}\text { Response rate of } \\
41.7 \% \text {; remission rate } \\
\text { of } 29.2 \%\end{array}$ & $\begin{array}{l}\text { Four serious } \\
\text { adverse events } \\
\text { reported, all having } \\
\text { to do with suicidal } \\
\text { ideation or } \\
\text { worsening } \\
\text { depressive } \\
\text { symptoms } \\
\text { determined } \\
\text { unrelated to rTMS } \\
\text { treatment }\end{array}$ \\
\hline $\begin{array}{l}\text { Croarkin et al. (7) } \\
\text { Neuropsychopharmacology } \\
\text { Sham arm }\end{array}$ & $n=55$ & $\begin{array}{l}M=17.4 \\
\text { Range = } \\
12-21\end{array}$ & $\begin{array}{l}18 \text { males, } \\
37 \text { females }\end{array}$ & Sham & $\begin{array}{l}30 \text { treatment } \\
\text { sessions over } 6 \\
\text { weeks }\end{array}$ & $\begin{array}{l}\text { HAM-D, MADRS, } \\
\text { CRS-R, } \\
\text { QIDS-A-SR, } \\
\text { CGI-S }\end{array}$ & $\begin{array}{l}\text { zaleplon, zolpidem, } \\
\text { zopiclone, or } \\
\text { lorazepam }\end{array}$ & $\begin{array}{l}\text { Hedges's gav }= \\
1.15, \text { Glass's } \Delta_{\text {pre }} \\
=1.53\end{array}$ & $\begin{array}{l}\text { Response rate of } \\
36.4 \% \text {; remission rate } \\
\text { of } 29.0 \%\end{array}$ & $\begin{array}{l}\text { One serious } \\
\text { adverse event of } \\
\text { suicidal ideation } \\
\text { definitely unrelated } \\
\text { to rTMS treatment }\end{array}$ \\
\hline
\end{tabular}




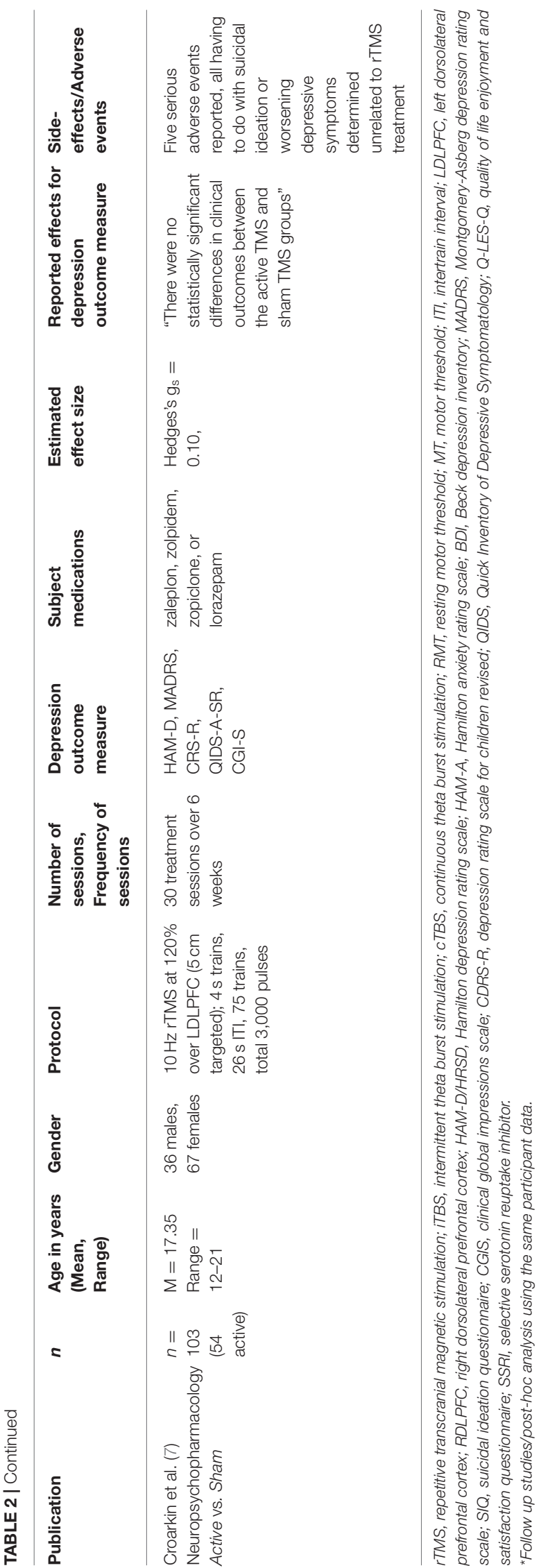

\section{LIMITATIONS OF THE EXISTING LITERATURE AND PROPOSED RESOLUTIONS}

\section{Impact of Stimulation Parameters on Effect Size}

The majority of clinical trials for both adult and adolescent depression have applied $10 \mathrm{~Hz}$ left hemisphere DLPFC rTMS. However, a meta-analysis of rTMS RCTs, encompassing over 4,000 patients and 81 trials, concluded that sequential bilateral stimulation to the right $(1 \mathrm{~Hz})$ and left $(10 \mathrm{~Hz})$ DLPFC was the most effective method (177). Only two adolescent depression trials used bilateral stimulation. Though safety and efficacy are the primary goals of novel treatment development, feasibility and tolerability also need to be considered. The standard $10 \mathrm{~Hz}$ protocol is burdensome for the patient and provider; daily sessions require over half an hour (up to an hour for bilateral stimulation protocols) and at least 6 weeks of treatment. Use of iTBS protocols can reduce this burden with a single session of iTBS taking only $3 \mathrm{~min}$, allowing for accelerated protocols and shorter treatment courses (62).

iTBS protocols were designed to closely mimic endogenous theta/gamma rhythms of the brain and induce long-term potentiation-like (LTP-like) plasticity. Though early iTBS studies targeted primary motor cortex, this protocol has also been applied to other brain regions including the DLPFC. iTBS over the DLPFC has been shown to induce long-term changes in local cortical excitability $(178,179)$, reduce GABA and glutamate/glutamine levels, and alter network connectivity (180). On a behavioral level, iTBS led to improved performance on the ability to inhibit automatic responses and working memory tasks in a small study of healthy volunteers (181). In a non-inferiority study iTBS and $10 \mathrm{~Hz}$ rTMS both reduced symptoms of depression in adults with similar safety, tolerability and efficacy (182). A meta-analysis of adult studies showed a response rate of iTBS of $35.6 \%$ (42/118) vs. $17.5 \%$ (18/103) with sham, a pooled odds ratio of response and remission of 2.7 and 1.9, respectively, and an effect size of 1.0 (183). Though promising in adults, iTBS has not been evaluated in an RCT in adolescents and faces the challenge of the aforementioned higher sham response rates. However, the mechanism of action of iTBS may be particularly well-suited to the intrinsic neuroplasticity of the adolescent brain. Furthermore, the ability of iTBS to modulate aberrant neurotransmitter function and pathological connectivity is well-matched to the reported DLPFC pathophysiology of adolescent depression.

Different rTMS targeting strategies yield differences in precision, reliability, and effect size. In six of 12 studies (Table 2) of adolescent depression the targeting strategy was scalp-based, four used MRI-guidance, and two provide no data. Scalp-based targeting is unreliable and imprecise for localizing DLPFC (184188). MRI-based neuronavigation used for rTMS shows larger effect sizes $(127,130,131)$. For treatment of depression, one study in 51 depressed adults found a moderately larger effect for MRI-based neuronavigation than standard targeting (Hedges $\left.\mathrm{g}_{\mathrm{s}}=0.64\right)(127)$. Even when using MRI-based neuronavigation, 
there are uncertainties about the optimal target for treating depression. Most studies target DLPFC, a large cortical area that is functionally connected to the Frontoparietal Network (also known as the Central Executive Network), the Default Mode Network, and the Salience Network (93, 189). All of these networks may be affected in depression and could be influenced by DLPFC stimulation. Using a standard "figure of 8 coil," a shift of as little as $0.5-1 \mathrm{~cm}$ can differentially affect one or more of these networks (190). Furthermore, there is a large variation in individual functional brain circuitry (191). Thus, especially during adolescence when these networks are in flux, using patient-specific functional neuronavigated rTMS to precisely target and modulate one or more of these networks, could lead to larger effect sizes (192).

Larger effect sizes are also seen in protocols that apply more sessions and more pulses per session (193-195). A meta-analysis of number of sessions and pulses/session to treat depression found the average effect size increased from 0.43 to 2.74 when the number of sessions increased from 5 to 20; the maximum mean effect size (5.47) was seen with 20 or more sessions and more than 1,200 pulses/session (193). However, only five of the 12 studies of adolescent depression applied 20 or more sessions. Increasing the number of sessions per day above the convention of one session daily would increase efficiency. The conventional procedure results in a standard course of treatment of 6 weeks or more. Accelerated protocols with 2-10 session per day applying the same number of total sessions appear to show equivalent safety and efficacy (157, 195-199) with the pace of improvement showing a direct relationship with the cumulative number of sessions. Additionally, neuroimaging studies find that, like standard protocols, accelerated protocols result in changes on neurochemical and functional connectivity biomarkers of depression $(103,200)$. When applying more than one session daily, intersession interval influences additive effects. Basic research studies on LTP find that the level of LTP is doubled when a second TBS train is applied after $60 \mathrm{~min}$, but if applied after only 10, 30, or $40 \mathrm{~min}$ there was no cumulative effect (201). That being said, neurodevelopmental factors could affect optimal intersession intervals and has yet to be determined. Piecing these protocol parameters together, a proposal for the most favorable balance of feasibility, efficacy and efficiency is up to five sessions/day, a 60-min intersession interval, and at least 20 total sessions (202). Such a treatment course can be completed in 1 week (instead of 6 weeks for once daily treatments). The value of swift antidepressant interventions becomes particularly relevant during the COVID pandemic, when access to therapy can be hindered and also adherence to longer therapy is a greater problem (203).

\section{Within-Group Variability: Impact of Inter-individual Heterogeneity and Intra-individual Brain State on Effect Size}

Clinical heterogeneity may contribute to reduced therapeutic response in studies of adolescents $(204,205)$. Adolescent patients present with a diverse range of symptoms (205), clinical courses (206), and responses to treatment $(16,204,207,208)$. We and others have thus suggested that underlying pathophysiology may account for the observed clinical heterogeneity (209). Given this heterogeneity, one would not expect to find a single "one size fits all" optimal treatment for adolescent depression (210). To address clinical heterogeneity, one could increase the sample size, allowing for subgroup analyses, or focus on a narrower phenotype.

Attempts have been made to define clinical subtypes and brain-network-based biotypes, but these have been difficult to replicate (211). One promising symptom domain for targeted treatment is anhedonia/dysphoria. Anhedonic/dysphoric symptoms seem to be reliably associated with PFC-cingulate network dysfunction (212-215) and have been shown to be particularly responsive to neuronavigated rTMS to the DLPFC (100). This raises the possibility that reducing sample heterogeneity by enrolling with primarily anhedonic/dysphoric symptoms might increase power to observe an effect. Such an approach was successfully used in a recent pharmacological trial (216).

A different approach is to focus treatment on suicidal ideation, the most serious risk to patient safety. The literature on efficacy of rTMS for suicidality paints a mixed picture. A pooled analysis of 19 depressed adolescents who received open-label rTMS showed decreased suicidal ideation over the course of treatment (153). This decrease in suicidality, corresponded to an overall decrease in severity of depression symptoms (153). A retrospective analysis of 332 depressed adult patients who received rTMS also reported improvements in suicidality (217) and a review $(n=593)$ and naturalistic study $(n=43)$ of rTMS in adult MDD found consistent improvements in depressive symptoms and suicidality in open-label trials. These positive findings are contrasted with results from sham-controlled trials that have failed to show significant group differences in the reduction of suicidal ideation (210). While it is possible that, compared to conventional pharmacotherapy, rTMS might be a safe, faster means of reducing suicidal ideation; larger, sham-controlled RCTs employing the most current sophisticated methods will be needed to conclusively demonstrate efficacy. Furthermore, the immediate therapeutic effects of ketamine may soon become the standard for speed of reducing suicidality against which all other interventions must be compared.

For both safety and feasibility, most adult and adolescent rTMS studies have allowed participants to continue their current medications (218). Safety reviews suggest that rTMS in those receiving stable doses of antidepressant medication does not increase the risk of adverse events (82); however, this increases within-group variability in neurochemical state and decreases statistical power. To control for variability in neurochemical state, investigators could enroll only participants who have been withdrawn from all psychotropic medications; however, withdrawing symptomatic patients from their medications introduces safety concerns of increased suicidal ideation and withdrawal-related side-effects. It demands close medical monitoring. A more feasible approach would be to require participants to maintain a steady medication dose and to apply a within-subject model controlling for baseline severity as the primary outcome measure. While this would not 
eliminate variability across participants, it reduces the effects of neurochemical brain state on the primary outcome measure. Combining rTMS and pharmacological treatment is another novel multimodal intervention being developed in adults that could be extended to adolescents (219). Notably, recent data suggest that combining antidepressant medication with a course of rTMS may in fact have a greater benefit in adolescents than in adults (150).

In order to increase tolerability of the treatment, adolescent studies have allowed patients to read, watch TV, or listen to music during rTMS sessions. The difference in behavioral engagement/arousal this causes is another potential source of within-group variability. While these distraction strategies are common in everyday practice with rTMS, they are a concern for treatment trials. Factors such as attention, arousal and mood state have been shown to affect modulation of excitability by rTMS (220-223). Thus, if the adolescent is even passively engaged in an unrelated activity, this may impact the effect of the rTMS. Applying shorter stimulation protocols (e.g., iTBS) may reduce the need for co-occurring activities to make the session more tolerable. Alternatively, one can transiently modify the patient's cognitive state by presenting stimuli or engage them in a task that engages the same brain networks as the rTMS target. In this way, rTMS can be combined with the behavioral task in order to amplify the impact on the targeted network (76). Studies of rTMS for post-traumatic stress disorder (PTSD), smoking cessation, and OCD have shown increased treatment response when the participant's symptoms were provoked [e.g., by asking questions about thoughts, images, or impulses related to their obsessions or compulsions or asking the patient to perform a task related to their symptoms (224)] immediately prior to rTMS stimulation (225-227). One could also consider pairing stimulation with concurrent behavioral interventions such as CBT (228).

\section{The Importance of Imaging Biomarkers in rTMS Treatment Development}

Given the significant time-investment necessary for rTMS treatment, many have sought to identify early predictors of later response. A recent retrospective study of 101 patients who received 4 weeks of rTMS treatment found that a lack of clinical response at the midway point predicted nonresponse with $88 \%$ accuracy (229). However, this still requires the patient to undergo 10 treatment sessions. Identifying intrinsic neurophysiological signatures or measures that can be obtained after one or two rTMS sessions would be preferable (229). Brain imaging techniques are increasingly capable of obtaining measures of brain function at cellular/molecular and network levels $(96,97,230,231)$. MRS can yield measures of GABA and glutamate neurotransmitter functioning (230, 231). BOLD fMRI and $\operatorname{RSFC}(96,97)$ have been proposed as tools for neuroimaging biomarkers. RSFC as measured by fMRI is stable across development $(232,233)$ and reproducible (234) at a group level. RSFC in adolescent depression has been shown to differentiate (at a group-, not at the individual-level) symptom severity (235), symptom domains
$(213,236,237)$, onset $(238)$, and course of disease $(239,240)$. Furthermore, RSFC in fronto-parietal, cingulo-opercular/ventral attention, and default mode networks has been used to predict individual differences in adolescent brain maturity and executive functioning (241). Within individuals, however, most commonly used sequences are too short to produce reliable RSFC measures (242), but with longer sequences $(243,244)$ or novel sequence types (244) it may be a reliable measure. rTMS trials in adolescent depression would benefit from dense collection of reliable neuroimaging measures at baseline, posttreatment, and follow-up. At baseline, neuroimaging measures may allow investigators to customize treatment to the unique neurodevelopmental state of each adolescent's developing cortex. Though group data appear promising, the benefit of using RSFC profiles for patient-specific treatment decisions is currently only theoretical.

Neuroimaging measures can also serve as predictive biomarkers for response to rTMS. Multiple studies have linked intrinsic functional connectivity between the targeted region of PFC and ACC, anterior insula and striatum with later response to $\operatorname{rTMS}(74,125,200,245-254)$ and other antidepressant treatments (101, 255-258). Interleaved rTMS/fMRI has shown acute effects on BOLD activation of both local and distal brain regions immediately following $1 \mathrm{~Hz}$ DLPFC rTMS in adults with MDD (89). Though research is promising in this area, replication and prospective examination of these predictive biomarkers will be critical prior to clinical implementation.

To facilitate clinical translation of such biomarkers, the National Institute of Mental Health (NIMH) has introduced "fast-fail" trials. This initiative employs an experimental therapeutics platform to quickly identify devices, protocols or compounds that should be considered for more extensive testing (259). These types of trials may be especially useful in adolescent depression where there is a clear urgent public health need for novel therapeutics, but still a great deal of uncertainty in the neural targets and biomarkers that reliably predict treatment response. The "fast-fail" initiative has recently led researchers to report successful target-engagement of a novel kappa-opioid receptor antagonist for the treatment of anhedonia in adult MDD (216). Thus, extending this approach to adolescents is both timely and feasible.

\section{CONCLUSIONS}

Treating adolescent depression is fraught with the challenges of heterogeneity of the clinical phenotype, the high placebo response rate, and the breadth of neurodevelopmental changes during puberty. While the efficacy of rTMS for adults with treatment resistant MDD is supported by multiple, adequately powered RCTs, evidence in adolescent depression is scanty and has many limitations. The safety profile of rTMS for adolescent depression appears to be desirable but many parameters, including the most favorable approaches, have yet to be determined. 
rTMS is worthy of further investigation for this vulnerable population. We propose that future adolescent depression rTMS trials: (1) be designed with 20 or more sessions (2) select a narrow clinical phenotype (e.g., select for anhedonia); (3) test for the possibility of individualization by including neuroimaging biomarkers (e.g., RSFC); (4) employ an experimental therapeutics approach (5) allow for robust inferences by being adequately controlled and powered with realistic effect size estimates. Such trials, if successful, may establish paradigms for larger, pivotal trials that clarify whether rTMS is an effective treatment for adolescent depression. Furthermore, they are likely to advance our understanding of the pathophysiology of this disorder.

\section{REFERENCES}

1. Hallett M. Transcranial magnetic stimulation: a primer. Neuron. (2007) 55:187-99. doi: 10.1016/j.neuron.2007.06.026

2. Croarkin PE, MacMaster FP. Transcranial magnetic stimulation for adolescent depression. Child Adolesc Psychiatr Clin N Am. (2019) 28:3343. doi: 10.1016/j.chc.2018.07.003

3. Donaldson AE, Gordon MS, Melvin GA, Barton DA, Fitzgerald PB. Addressing the needs of adolescents with treatment resistant depressive disorders: a systematic review of rTMS. Brain Stimul. (2014) 7:712. doi: 10.1016/j.brs.2013.09.012

4. Magavi LR, Reti IM, Vasa RA. A review of repetitive transcranial magnetic stimulation for adolescents with treatment-resistant depression. Int Rev Psychiatry. (2017) 29:79-88. doi: 10.1080/09540261.2017.1300574

5. Hett D, Rogers J, Humpston C, Marwaha S. Repetitive transcranial magnetic stimulation (rtms) for the treatment of depression in adolescence: a systematic review. J Affect Disord. (2020) 278:460-9. doi: 10.1016/j.jad.2020.09.058

6. Croarkin PE, Wall CA, McClintock SM, Kozel FA, Husain MM, Sampson SM. The emerging role for repetitive transcranial magnetic stimulation in optimizing the treatment of adolescent depression. J ECT. (2010) 26:3239. doi: 10.1097/YCT.0b013e3181dd17eb

7. Croarkin PE, Elmaadawi AZ, Aaronson ST, Schrodt GR Jr, Holbert RC, Verdoliva S, et al. Left prefrontal transcranial magnetic stimulation for treatment-resistant depression in adolescents: a double-blind, randomized, sham-controlled trial. Neuropsychopharmacology. (2020) 46:462-9. doi: 10.1038/s41386-02000829-y

8. Le K, Liu L, Sun M, Hu L, Xiao N. Transcranial magnetic stimulation at 1 Hertz improves clinical symptoms in children with Tourette syndrome for at least 6 months. J Clin Neurosci. (2013) 20:25762. doi: 10.1016/j.jocn.2012.01.049

9. Cristancho P, Akkineni K, Constantino JN, Carter AR, O'Reardon JP. Transcranial magnetic stimulation in a 15-year-old patient with autism and comorbid depression. J ECT. (2014) 30:e467. doi: 10.1097/YCT.0000000000000156

10. Friedrich MJ. Depression is the leading cause of disability around the world. JAMA. (2017) 317:1517. doi: 10.1001/jama.2017.3826

11. Disease GBD, Injury I, Prevalence C. Global, regional, and national incidence, prevalence, and years lived with disability for 328 diseases and injuries for 195 countries, 1990-2016: a systematic analysis for the Global Burden of Disease Study 2016. Lancet. (2017) 390:121159. doi: 10.1016/S0140-6736(17)32154-2

12. Pratt LA, Brody DJ. Depression in the U.S. household population, 2009-2012. NCHS Data Brief. (2014) 1-8.

13. Costello EJ, Mustillo S, Erkanli A, Keeler G, Angold A. Prevalence and development of psychiatric disorders in childhood and adolescence. Arch Gen Psychiatry. (2003) 60:837-44. doi: 10.1001/archpsyc.60.8.837

\section{AUTHOR CONTRIBUTIONS}

LO drafted and revised the manuscript. $\mathrm{MH}$ conducted the literature review under the supervision of LO. DN, KT, SL, and AS contributed to the drafting and revision of the manuscript. All authors approved the final submitted manuscript.

\section{FUNDING}

This research was supported in part by the Intramural Research Program of the National Institute of Mental Health National Institutes of Health (NIH) (Grant No. ZIA-MH002957-01 [to AS] and ZIA-MH002955-04 [to SL]).

14. Brent DA, Perper JA, Moritz G, Allman C, Friend A, Roth C, et al. Psychiatric risk factors for adolescent suicide: a casecontrol study. J Am Acad Child Adolesc Psychiatry. (1993) 32:521-9. doi: 10.1097/00004583-199305000-00006

15. Turecki G, Brent DA. Suicide and suicidal behaviour. Lancet. (2016) 387:1227-39. doi: 10.1016/S0140-6736(15)00234-2

16. Cuijpers P, Stringaris A, Wolpert M. Treatment outcomes for depression: challenges and opportunities. Lancet Psychiatry. (2020) 7:925-7. doi: 10.1016/S2215-0366(20)30036-5

17. Dwyer JB, Stringaris A, Brent DA, Bloch MH. Annual research review: defining and treating pediatric treatment-resistant depression. J Child Psychol Psychiatry. (2020) 61:312-32. doi: 10.1111/jcpp. 13202

18. Kennard B, Silva S, Vitiello B, Curry J, Kratochvil C, Simons $A$, et al. Remission and residual symptoms after short-term treatment in the Treatment of Adolescents with Depression Study (TADS). J Am Acad Child Adolesc Psychiatry. (2006) 45:1404-11. doi: 10.1097/01.chi.0000242228.75516.21

19. Kennard BD, Emslie GJ, Mayes TL, Hughes JL. Relapse and recurrence in pediatric depression. Child Adolesc Psychiatr Clin N Am. (2006) 15:1057-79, xi. doi: 10.1016/j.chc.2006.05.003

20. Emslie GJ, Kennard BD, Mayes TL, Nightingale-Teresi J, Carmody T, Hughes CW, et al. Fluoxetine versus placebo in preventing relapse of major depression in children and adolescents. Am J Psychiatry. (2008) 165:45967. doi: 10.1176/appi.ajp.2007.07091453

21. Birmaher B, Brent D, Issues AWGoQ, Bernet W, Bukstein O, Walter H, et al. Practice parameter for the assessment and treatment of children and adolescents with depressive disorders. J Am Acad Child Adolesc Psychiatry. (2007) 46:1503-26. doi: 10.1097/chi.0b013e318145ae1c

22. Konig $\mathrm{H}$, Konig $\mathrm{HH}$, Konnopka A. The excess costs of depression: a systematic review and meta-analysis. Epidemiol Psychiatr Sci. (2019) 29:e30. doi: 10.1017/S2045796019000180

23. Voigt J, Carpenter L, Leuchter A. Cost effectiveness analysis comparing repetitive transcranial magnetic stimulation to antidepressant medications after a first treatment failure for major depressive disorder in newly diagnosed patients - a lifetime analysis. PLoS ONE. (2017) 12:e186950. doi: 10.1371/journal.pone.0186950

24. Beesdo K, Hofler M, Leibenluft E, Lieb R, Bauer M, Pfennig A. Mood episodes and mood disorders: patterns of incidence and conversion in the first three decades of life. Bipolar Disord. (2009) 11:63749. doi: 10.1111/j.1399-5618.2009.00738.x

25. Hankin BL. Depression during childhood and adolescence. In: Derubeis RJ, Strunk DR, editors. Oxford Handbook of Mood Disorders. New York, NY: Oxford University Press (2017). p. 276-86. doi: 10.1093/oxfordhb/9780199973965.013.24

26. Gan WB, Kwon E, Feng G, Sanes JR, Lichtman JW. Synaptic dynamism measured over minutes to months: age-dependent decline in an autonomic ganglion. Nat Neurosci. (2003) 6:956-60. doi: 10.1038/nn1115 
27. Zuo Y, Lin A, Chang P, Gan WB. Development of long-term dendritic spine stability in diverse regions of cerebral cortex. Neuron. (2005) 46:1819. doi: 10.1016/j.neuron.2005.04.001

28. Casey B, Jones RM, Somerville LH. Braking and accelerating of the adolescent brain. $J$ Res Adolesc. (2011) 21:2133. doi: 10.1111/j.1532-7795.2010.00712.x

29. Ernst M, Pine DS, Hardin M. Triadic model of the neurobiology of motivated behavior in adolescence. Psychol Med. (2006) 36:299312. doi: 10.1017/S0033291705005891

30. Lewis DA. Development of the prefrontal cortex during adolescence: insights into vulnerable neural circuits in schizophrenia. Neuropsychopharmacology. (1997) 16:385-98. doi: 10.1016/S0893-133X(96)00277-1

31. Sowell ER, Peterson BS, Thompson PM, Welcome SE, Henkenius AL, Toga AW. Mapping cortical change across the human life span. Nat Neurosci. (2003) 6:309-15. doi: 10.1038/nn1008

32. Luna B, Garver KE, Urban TA, Lazar NA, Sweeney JA. Maturation of cognitive processes from late childhood to adulthood. Child Dev. (2004) 75:1357-72. doi: 10.1111/j.1467-8624.2004. 00745.x

33. Gogtay N, Thompson PM. Mapping gray matter development: implications for typical development and vulnerability to psychopathology. Brain Cogn. (2010) 72:6-15. doi: 10.1016/j.bandc.2009. 08.009

34. Gogtay N, Giedd JN, Lusk L, Hayashi KM, Greenstein D, Vaituzis AC, et al. Dynamic mapping of human cortical development during childhood through early adulthood. Proc Natl Acad Sci USA. (2004) 101:81749. doi: 10.1073/pnas.0402680101

35. Giedd JN, Blumenthal J, Jeffries NO, Castellanos FX, Liu H, Zijdenbos A, et al. Brain development during childhood and adolescence: a longitudinal MRI study. Nat Neurosci. (1999) 2:861-3. doi: 10.1038/13158

36. Lenroot RK, Gogtay N, Greenstein DK, Wells EM, Wallace GL, Clasen LS, et al. Sexual dimorphism of brain developmental trajectories during childhood and adolescence. Neuroimage. (2007) 36:1065-73. doi: 10.1016/j.neuroimage.2007.03.053

37. Lebel C, Beaulieu C. Longitudinal development of human brain wiring continues from childhood into adulthood. J Neurosci. (2011) 31:1093747. doi: 10.1523/JNEUROSCI.5302-10.2011

38. Hasan KM, Sankar A, Halphen C, Kramer LA, Brandt ME, Juranek J, et al. Development and organization of the human brain tissue compartments across the lifespan using diffusion tensor imaging. Neuroreport. (2007) 18:1735-9. doi: 10.1097/WNR.0b013e3282f0d40c

39. Liston C, Watts R, Tottenham N, Davidson MC, Niogi S, Ulug AM, et al. Frontostriatal microstructure modulates efficient recruitment of cognitive control. Cereb Cortex. (2006) 16:553-60. doi: 10.1093/cercor/bhj003

40. Liston C, McEwen BS, Casey BJ. Psychosocial stress reversibly disrupts prefrontal processing and attentional control. Proc Natl Acad Sci USA. (2009) 106:912-7. doi: 10.1073/pnas.0807041106

41. Durston S, Davidson MC, Tottenham N, Galvan A, Spicer J, Fossella JA, et al. A shift from diffuse to focal cortical activity with development. Dev Sci. (2006) 9:1-8. doi: 10.1111/j.1467-7687.2005.00454.x

42. Casey BJ, Trainor RJ, Orendi JL, Schubert AB, Nystrom LE, Giedd JN, et al. A developmental functional MRI study of prefrontal activation during performance of a go-no-go task. J Cogn Neurosci. (1997) 9:83547. doi: 10.1162/jocn.1997.9.6.835

43. Stevens MC, Kiehl KA, Pearlson GD, Calhoun VD. Functional neural networks underlying response inhibition in adolescents and adults. Behav Brain Res. (2007) 181:12-22. doi: 10.1016/j.bbr.2007. 03.023

44. Casey BJ, Getz S, Galvan A. The adolescent brain. Dev Rev. (2008) 28:6277. doi: 10.1016/j.dr.2007.08.003

45. Thomases DR, Cass DK, Tseng KY. Periadolescent exposure to the NMDA receptor antagonist MK-801 impairs the functional maturation of local GABAergic circuits in the adult prefrontal cortex. J Neurosci. (2013) 33:2634. doi: 10.1523/JNEUROSCI.4147-12.2013

46. Hedner T, Iversen K, Lundborg P. Central GABA mechanisms during postnatal development in the rat: neurochemical characteristics. J Neural Transm. (1984) 59:105-18. doi: 10.1007/BF01255409
47. Wang HX, Gao WJ. Cell type-specific development of NMDA receptors in the interneurons of rat prefrontal cortex. Neuropsychopharmacology. (2009) 34:2028-40. doi: 10.1038/npp.2009.20

48. Ernst M, Daniele T, Frantz K. New perspectives on adolescent motivated behavior: attention and conditioning. Dev Cogn Neurosci. (2011) 1:37789. doi: 10.1016/j.dcn.2011.07.013

49. Wahlstrom D, White T, Luciana M. Neurobehavioral evidence for changes in dopamine system activity during adolescence. Neurosci Biobehav Rev. (2010) 34:631-48. doi: 10.1016/j.neubiorev.2009.12.007

50. Caballero A, Granberg R, Tseng KY. Mechanisms contributing to prefrontal cortex maturation during adolescence. Neurosci Biobehav Rev. (2016) 70:412. doi: 10.1016/j.neubiorev.2016.05.013

51. Chambers RA, Taylor JR, Potenza MN. Developmental neurocircuitry of motivation in adolescence: a critical period of addiction vulnerability. Am J Psychiatry. (2003) 160:1041-52. doi: 10.1176/appi.ajp.160.6.1041

52. O'Donnell P. Adolescent maturation of cortical dopamine. Neurotox Res. (2010) 18:306-12. doi: 10.1007/s12640-010-9157-3

53. Tseng KY, O’Donnell P. Dopamine modulation of prefrontal cortical interneurons changes during adolescence. Cereb Cortex. (2007) 17:123540. doi: 10.1093/cercor/bhl034

54. Sturman DA, Moghaddam B. The neurobiology of adolescence: changes in brain architecture, functional dynamics, and behavioral tendencies. Neurosci Biobehav Rev. (2011) 35:1704-12. doi: 10.1016/j.neubiorev.2011.04.003

55. Duman RS, Sanacora G, Krystal JH. Altered connectivity in depression: GABA and glutamate neurotransmitter deficits and reversal by novel treatments. Neuron. (2019) 102:75-90. doi: 10.1016/j.neuron.2019.03.013

56. Gabbay V, Mao X, Klein RG, Ely BA, Babb JS, Panzer AM, et al. Anterior cingulate cortex gamma-aminobutyric acid in depressed adolescents: relationship to anhedonia. Arch Gen Psychiatry. (2012) 69:139-49. doi: 10.1001/archgenpsychiatry.2011.131

57. Dubin MJ, Mao X, Banerjee S, Goodman Z, Lapidus KA, Kang $\mathrm{G}$, et al. Elevated prefrontal cortex GABA in patients with major depressive disorder after TMS treatment measured with proton magnetic resonance spectroscopy. J Psychiatry Neurosci. (2016) 41:E37-45. doi: 10.1503/jpn.150223

58. Croarkin PE, Nakonezny PA, Wall CA, Murphy LL, Sampson SM, Frye MA, et al. Transcranial magnetic stimulation potentiates glutamatergic neurotransmission in depressed adolescents. Psychiatry Res Neuroimaging. (2016) 247:25-33. doi: 10.1016/j.pscychresns.2015.11.005

59. Chen R, Cros D, Curra A, Di Lazzaro V, Lefaucheur JP, Magistris MR, et al. The clinical diagnostic utility of transcranial magnetic stimulation: report of an IFCN committee. Clin Neurophysiol. (2008) 119:50432. doi: 10.1016/j.clinph.2007.10.014

60. Rothwell JC. Paired-pulse investigations of short-latency intracortical facilitation using TMS in humans. Electroencephalogr Clin Neurophysiol Suppl. (1999) 51:113-9.

61. Kujirai T, Caramia MD, Rothwell JC, Day BL, Thompson PD, Ferbert A, et al. Corticocortical inhibition in human motor cortex. J Physiol. (1993) 471:501-19. doi: 10.1113/jphysiol.1993.sp019912

62. Huang YZ, Edwards MJ, Rounis E, Bhatia KP, Rothwell JC. Theta burst stimulation of the human motor cortex. Neuron. (2005) 45:2016. doi: 10.1016/j.neuron.2004.12.033

63. Hamada M, Ugawa Y. Quadripulse stimulation-a new patterned rTMS. Restor Neurol Neurosci. (2010) 28:419-24. doi: 10.3233/RNN-2010-0564

64. Hallett M. Transcranial magnetic stimulation and the human brain. Nature. (2000) 406:147-50. doi: 10.1038/35018000

65. Aleman A. Use of repetitive transcranial magnetic stimulation for treatment in psychiatry. Clin Psychopharmacol Neurosci. (2013) 11:539. doi: 10.9758/cpn.2013.11.2.53

66. Pascual-Leone A, Rubio B, Pallardo F, Catala MD. Rapid-rate transcranial magnetic stimulation of left dorsolateral prefrontal cortex in drug-resistant depression. Lancet. (1996) 348:233-7. doi: 10.1016/S0140-6736(96)01219-6

67. Nahas Z, Lomarev M, Roberts DR, Shastri A, Lorberbaum JP, Teneback C, et al. Unilateral left prefrontal transcranial magnetic stimulation (TMS) produces intensity-dependent bilateral effects as measured by interleaved BOLD fMRI. Biol Psychiatry. (2001) 50:712-20. doi: 10.1016/S0006-3223(01)01199-4 
68. Kimbrell TA, Little JT, Dunn RT, Frye MA, Greenberg BD, Wassermann EM, et al. Frequency dependence of antidepressant response to left prefrontal repetitive transcranial magnetic stimulation (rTMS) as a function of baseline cerebral glucose metabolism. Biol Psychiatry. (1999) 46:160313. doi: $10.1016 /$ S0006-3223(99)00195-X

69. Fitzgerald PB, Sritharan A, Daskalakis ZJ, de Castella AR, Kulkarni J, Egan G. A functional magnetic resonance imaging study of the effects of low frequency right prefrontal transcranial magnetic stimulation in depression. J Clin Psychopharmacol. (2007) 27:488-92. doi: 10.1097/jcp.0b013e318151521c

70. Beynel L, Powers JP, Appelbaum LG. Effects of repetitive transcranial magnetic stimulation on resting-state connectivity: a systematic review. Neuroimage. (2020) 211:116596. doi: 10.1016/j.neuroimage.2020.116596

71. Hawco C, Voineskos AN, Steeves JKE, Dickie EW, Viviano JD, Downar J, et al. Spread of activity following TMS is related to intrinsic resting connectivity to the salience network: a concurrent TMS-fMRI study. Cortex. (2018) 108:160-72. doi: 10.1016/j.cortex.2018.07.010

72. Tang Y, Jiao X, Wang J, Zhu T, Zhou J, Qian Z, et al. Dynamic functional connectivity within the fronto-limbic network induced by intermittent theta-burst stimulation: a pilot study. Front Neurosci. (2019) 13:944. doi: 10.3389/fnins.2019.00944

73. Alkhasli I, Sakreida K, Mottaghy FM, Binkofski F. Modulation of frontostriatal functional connectivity using transcranial magnetic stimulation. Front Hum Neurosci. (2019) 13:190. doi: 10.3389/fnhum.2019.00190

74. Fox MD, Buckner RL, White MP, Greicius MD, Pascual-Leone A. Efficacy of transcranial magnetic stimulation targets for depression is related to intrinsic functional connectivity with the subgenual cingulate. Biol Psychiatry. (2012) 72:595-603. doi: 10.1016/j.biopsych.2012.04.028

75. Silvanto J, Pascual-Leone A. State-dependency of transcranial magnetic stimulation. Brain Topogr. (2008) 21:1-10. doi: 10.1007/s10548-008-0067-0

76. Schiena G, Maggioni E, Pozzoli S, Brambilla P. Transcranial magnetic stimulation in major depressive disorder: response modulation and state dependency. J Affect Disord. (2020) 266:793-801. doi: 10.1016/j.jad.2020.02.006

77. Machii K, Cohen D, Ramos-Estebanez C, Pascual-Leone A. Safety of rTMS to non-motor cortical areas in healthy participants and patients. Clin Neurophysiol. (2006) 117:455-71. doi: 10.1016/j.clinph.2005.10.014

78. Loo CK, McFarquhar TF, Mitchell PB. A review of the safety of repetitive transcranial magnetic stimulation as a clinical treatment for depression. Int $J$ Neuropsychopharmacol. (2008) 11:131-47. doi: 10.1017/S1461145707007717

79. Janicak PG, O'Reardon JP, Sampson SM, Husain MM, Lisanby SH, Rado JT, et al. Transcranial magnetic stimulation in the treatment of major depressive disorder: a comprehensive summary of safety experience from acute exposure, extended exposure, and during reintroduction treatment. $J$ Clin Psychiatry. (2008) 69:222-32. doi: 10.4088/JCP.v69n0208

80. Dobek CE, Blumberger DM, Downar J, Daskalakis ZJ, Vila-Rodriguez F. Risk of seizures in transcranial magnetic stimulation: a clinical review to inform consent process focused on bupropion. Neuropsychiatr Dis Treat. (2015) 11:2975-87. doi: 10.2147/NDT.S91126

81. Wassermann EM. Risk and safety of repetitive transcranial magnetic stimulation: report and suggested guidelines from the International Workshop on the Safety of Repetitive Transcranial Magnetic Stimulation, June 5-7, 1996. Electroencephalogr Clin Neurophysiol. (1998) 108:116. doi: 10.1016/S0168-5597(97)00096-8

82. Rossi S, Antal A, Bestmann S, Bikson M, Brewer C, Brockmöller J, et al. Safety and recommendations for TMS use in healthy subjects and patient populations, with updates on training, ethical and regulatory issues: expert guidelines. J Clin Neurophysiol. (2020) 132:269-306. doi: 10.1016/j.clinph.2020.10.003

83. Rossi S, Hallett M, Rossini PM, Pascual-Leone A, Safety of TMSCG. Safety, ethical considerations, and application guidelines for the use of transcranial magnetic stimulation in clinical practice and research. Clin Neurophysiol. (2009) 120:2008-39. doi: 10.1016/j.clinph.2009.08.016

84. Lerner AJ, Wassermann EM, Tamir DI. Seizures from transcranial magnetic stimulation 2012-2016: results of a survey of active laboratories and clinics. Clin Neurophysiol. (2019) 130:1409-16. doi: 10.1016/j.clinph.2019.03.016

85. George MS, Ketter TA, Post RM. Prefrontal cortex dysfunction in clinical depression. Depression. (1994) 2:59-72. doi: 10.1002/depr.3050020202
86. George MS, Wassermann EM, Williams WA, Callahan A, Ketter TA, Basser P, et al. Daily repetitive transcranial magnetic stimulation (rTMS) improves mood in depression. Neuroreport. (1995) 6:18536. doi: 10.1097/00001756-199510020-00008

87. George MS, Wassermann EM, Kimbrell TA, Little JT, Williams WE, Danielson AL, et al. Mood improvement following daily left prefrontal repetitive transcranial magnetic stimulation in patients with depression: a placebo-controlled crossover trial. Am J Psychiatry. (1997) 154:17526. doi: 10.1176/ajp.154.12.1752

88. George MS. Why would you ever want to? Toward understanding the antidepressant effect of prefrontal rTMS. Hum Psychopharmacol. (1998) 15:307-13. doi: 10.1002/(SICI)1099-1077(199807)13:5<307::AIDHUP1>3.0. CO;2-9

89. Li X, Nahas Z, Kozel FA, Anderson B, Bohning DE, George MS. Acute left prefrontal transcranial magnetic stimulation in depressed patients is associated with immediately increased activity in prefrontal cortical as well as subcortical regions. Biol Psychiatry. (2004) 55:88290. doi: 10.1016/j.biopsych.2004.01.017

90. Persson J, Struckmann W, Gingnell M, Fallmar D, Boden R. Intermittent theta burst stimulation over the dorsomedial prefrontal cortex modulates resting-state connectivity in depressive patients: a sham-controlled study. Behav Brain Res. (2020) 394:112834. doi: 10.1016/j.bbr.2020.112834

91. Tik M, Hoffmann A, Sladky R, Tomova L, Hummer A, Navarro de Lara $\mathrm{L}$, et al. Towards understanding rTMS mechanism of action: Stimulation of the DLPFC causes network-specific increase in functional connectivity. Neuroimage. (2017) 162:289-96. doi: 10.1016/j.neuroimage.2017.09.022

92. Fox MD, Halko MA, Eldaief MC, Pascual-Leone A. Measuring and manipulating brain connectivity with resting state functional connectivity magnetic resonance imaging (fcMRI) and transcranial magnetic stimulation (TMS). Neuroimage. (2012) 62:223243. doi: 10.1016/j.neuroimage.2012.03.035

93. Anderson RJ, Hoy KE, Daskalakis ZJ, Fitzgerald PB. Repetitive transcranial magnetic stimulation for treatment resistant depression: re-establishing connections. Clin Neurophysiol. (2016) 127:3394-405. doi: 10.1016/j.clinph.2016.08.015

94. Liston C, Chen AC, Zebley BD, Drysdale AT, Gordon R, Leuchter B, et al. Default mode network mechanisms of transcranial magnetic stimulation in depression. Biol Psychiatry. (2014) 76:517-26. doi: 10.1016/j.biopsych.2014.01.023

95. George MS, Short EB, Kerns SE. Brain stimulation treatments for depression. In: Derubeis RJ, Strunk DR, editors. Oxford Handbook of Mood Disorders. New York: Oxford University Press (2017). p. 398-410. doi: 10.1093/oxfordhb/9780199973965. 013.34

96. Glasser MF, Smith SM, Marcus DS, Andersson JL, Auerbach EJ, Behrens TE, et al. The human connectome project's neuroimaging approach. Nat Neurosci. (2016) 19:1175-87. doi: 10.1038/nn.4361

97. Jbabdi S, Sotiropoulos SN, Haber SN, Van Essen DC, Behrens TE. Measuring macroscopic brain connections in vivo. Nat Neurosci. (2015) 18:154655. doi: $10.1038 / \mathrm{nn} .4134$

98. Downar J, Geraci J, Salomons TV, Dunlop K, Wheeler S, McAndrews MP, et al. Anhedonia and reward-circuit connectivity distinguish nonresponders from responders to dorsomedial prefrontal repetitive transcranial magnetic stimulation in major depression. Biol Psychiatry. (2014) 76:176-85. doi: 10.1016/j.biopsych.2013.10.026

99. Drysdale AT, Grosenick L, Downar J, Dunlop K, Mansouri F, Meng Y, et al. Resting-state connectivity biomarkers define neurophysiological subtypes of depression. Nat Med. (2017) 23:28-38. doi: 10.1038/nm.4246

100. Siddiqi SH, Taylor SF, Cooke D, Pascual-Leone A, George MS, Fox MD. Distinct symptom-specific treatment targets for circuit-based neuromodulation. Am J Psychiatry. (2020) 177:435-46. doi: 10.1176/appi.ajp.2019.19090915

101. Williams LM. Defining biotypes for depression and anxiety based on large-scale circuit dysfunction: a theoretical review of the evidence and future directions for clinical translation. Depress Anxiety. (2017) 34:924. doi: 10.1002/da.22556

102. Croarkin PE, Nakonezny PA, Husain MM, Melton T, Buyukdura JS, Kennard $\mathrm{BD}$, et al. Evidence for increased glutamatergic cortical facilitation in children 
and adolescents with major depressive disorder. JAMA Psychiatry. (2013) 70:291-9. doi: 10.1001/2013.jamapsychiatry.24

103. Baeken C, Lefaucheur JP, Van Schuerbeek P. The impact of accelerated high frequency rTMS on brain neurochemicals in treatment-resistant depression: insights from (1)H MR spectroscopy. Clin Neurophysiol. (2017) 128:166472. doi: 10.1016/j.clinph.2017.06.243

104. Luborzewski A, Schubert F, Seifert F, Danker-Hopfe H, Brakemeier EL, Schlattmann P, et al. Metabolic alterations in the dorsolateral prefrontal cortex after treatment with high-frequency repetitive transcranial magnetic stimulation in patients with unipolar major depression. J Psychiatr Res. (2007) 41:606-15. doi: 10.1016/j.jpsychires.2006.02.003

105. Vidal-Pineiro D, Martin-Trias P, Falcon C, Bargallo N, Clemente IC, VallsSole J, et al. Neurochemical modulation in posteromedial default-mode network cortex induced by transcranial magnetic stimulation. Brain Stimul. (2015) 8:937-44. doi: 10.1016/j.brs.2015.04.005

106. Yang XR, Kirton A, Wilkes TC, Pradhan S, Liu I, Jaworska N, et al. Glutamate alterations associated with transcranial magnetic stimulation in youth depression: a case series. J ECT. (2014) 30:242-7. doi: 10.1097/YCT.0000000000000094

107. Allen CH, Kluger BM, Buard I. Safety of transcranial magnetic stimulation in children: a systematic review of the literature. Pediatr Neurol. (2017) 68:3-17. doi: 10.1016/j.pediatrneurol.2016.12.009

108. Zewdie E, Ciechanski P, Kuo HC, Giuffre A, Kahl C, King R, et al. Safety and tolerability of transcranial magnetic and direct current stimulation in children: prospective single center evidence from 3.5 million stimulations. Brain Stimul. (2020) 13:565-75. doi: 10.1016/j.brs.2019.12.025

109. Krishnan C, Santos L, Peterson MD, Ehinger M. Safety of noninvasive brain stimulation in children and adolescents. Brain Stimul. (2015) 8:7687. doi: $10.1016 /$ j.brs.2014.10.012

110. Hong YH, Wu SW, Pedapati EV, Horn PS, Huddleston DA, Laue CS, et al. Safety and tolerability of theta burst stimulation vs. single and paired pulse transcranial magnetic stimulation: a comparative study of 165 pediatric subjects. Front Hum Neurosci. (2015) 9:29. doi: 10.3389/fnhum.2015.00029

111. Hu SH, Wang SS, Zhang MM, Wang JW, Hu JB, Huang ML, et al. Repetitive transcranial magnetic stimulation-induced seizure of a patient with adolescent-onset depression: a case report and literature review. J Int Med Res. (2011) 39:2039-44. doi: 10.1177/147323001103900552

112. Chiramberro M, Lindberg N, Isometsa E, Kahkonen S, Appelberg B. Repetitive transcranial magnetic stimulation induced seizures in an adolescent patient with major depression: a case report. Brain Stimul. (2013) 6:830-1. doi: 10.1016/j.brs.2013.02.003

113. Cullen KR, Jasberg S, Nelson B, Klimes-Dougan B, Lim KO, Croarkin PE. Seizure induced by deep transcranial magnetic stimulation in an adolescent with depression. J Child Adolesc Psychopharmacol. (2016) 26:63741. doi: 10.1089/cap.2016.0070

114. Wang T, Huang L, Xu H, Yan L, Wu Q, Shen F, et al. Seizure induced by repetitive transcranial magnetic stimulation in an adolescent with migraine with aura. Brain Stimul. (2018) 11:1380-1. doi: 10.1016/j.brs.2018.07.052

115. Purushotham A, Sinha VK, Goyal N, Tikka SK. Intermittent theta burst stimulation induced seizure in a child with schizophrenia: a case report. Brain Stimul. (2018) 11:1415-6. doi: 10.1016/j.brs.2018.09.008

116. Kallel L, Brunelin J. A case report of transcranial magnetic stimulationrelated seizure in a young patient with major depressive disorder receiving accelerated transcranial magnetic stimulation. J ECT. (2020) 36:e31e2. doi: 10.1097/YCT.0000000000000666

117. Oberman LM, Enticott P (editors.). Neurotechnology and Brain Stimulation in Pediatric Psychiatric and Neurodevelopmental Disorders. 1st Edition. London: Elsevier (2019).

118. Kirton A, Gilbert DL (editors.). Pediatric Brain Stimulation: Mapping and Modulating the Developing Brain. London: Elsevier (2016).

119. Lefaucheur JP, Aleman A, Baeken C, Benninger DH, Brunelin J, Di Lazzaro $\mathrm{V}$, et al. Evidence-based guidelines on the therapeutic use of repetitive transcranial magnetic stimulation (rTMS): an update (2014-2018). Clin Neurophysiol. (2020) 131:474-528. doi: 10.1016/j.clinph.2020.02.003

120. Lefaucheur JP, Andre-Obadia N, Antal A, Ayache SS, Baeken C, Benninger $\mathrm{DH}$, et al. Evidence-based guidelines on the therapeutic use of repetitive transcranial magnetic stimulation (rTMS). Clin Neurophysiol. (2014) 125:2150-206. doi: 10.1016/j.clinph.2014.05.021
121. Avery DH, Isenberg KE, Sampson SM, Janicak PG, Lisanby SH, Maixner $\mathrm{DF}$, et al. Transcranial magnetic stimulation in the acute treatment of major depressive disorder: clinical response in an open-label extension trial. J Clin Psychiatry. (2008) 69:441-51. doi: 10.4088/JCP.v69n0315

122. Lisanby SH, Husain MM, Rosenquist PB, Maixner D, Gutierrez R, Krystal A, et al. Daily left prefrontal repetitive transcranial magnetic stimulation in the acute treatment of major depression: clinical predictors of outcome in a multisite, randomized controlled clinical trial. Neuropsychopharmacology. (2009) 34:522-34. doi: 10.1038/npp.2008.118

123. O’Reardon JP, Solvason HB, Janicak PG, Sampson S, Isenberg KE, Nahas Z, et al. Efficacy and safety of transcranial magnetic stimulation in the acute treatment of major depression: a multisite randomized controlled trial. Biol Psychiatry. (2007) 62:1208-16. doi: 10.1016/j.biopsych.2007.01.018

124. McClintock SM, Reti IM, Carpenter LL, McDonald WM, Dubin M, Taylor SF, et al. Consensus recommendations for the clinical application of repetitive Transcranial Magnetic Stimulation (rTMS) in the treatment of depression. $J$ Clin Psychiatry. (2018) 79:16cs10905. doi: 10.4088/JCP.16cs10905

125. Fox MD, Liu H, Pascual-Leone A. Identification of reproducible individualized targets for treatment of depression with TMS based on intrinsic connectivity. Neuroimage. (2013) 66:15160. doi: 10.1016/j.neuroimage.2012.10.082

126. Connolly KR, Helmer A, Cristancho MA, Cristancho P, O’Reardon JP. Effectiveness of transcranial magnetic stimulation in clinical practice postFDA approval in the United States: results observed with the first 100 consecutive cases of depression at an academic medical center. J Clin Psychiatry. (2012) 73:e567-73. doi: 10.4088/JCP.11m07413

127. Fitzgerald PB, Hoy K, McQueen S, Maller JJ, Herring S, Segrave R, et al. A randomized trial of rTMS targeted with MRI based neuro-navigation in treatment-resistant depression. Neuropsychopharmacology. (2009) 34:125562. doi: 10.1038/npp.2008.233

128. Gordon EM, Laumann TO, Gilmore AW, Newbold DJ, Greene DJ, Berg JJ, et al. Precision functional mapping of individual human brains. Neuron. (2017) 95:791-807 e7. doi: 10.1016/j.neuron.2017.07.011

129. Laumann TO, Gordon EM, Adeyemo B, Snyder AZ, Joo SJ, Chen MY, et al. Functional system and areal organization of a highly sampled individual human brain. Neuron. (2015) 87:657-70. doi: 10.1016/j.neuron.2015.06.037

130. Sack AT, Cohen Kadosh R, Schuhmann T, Moerel M, Walsh V, Goebel R. Optimizing functional accuracy of TMS in cognitive studies: a comparison of methods. J Cogn Neurosci. (2009) 21:207-21. doi: 10.1162/jocn.2009.21126

131. Beynel L, Appelbaum LG, Luber B, Crowell CA, Hilbig SA, Lim W, et al. Effects of online repetitive transcranial magnetic stimulation (rTMS) on cognitive processing: a meta-analysis and recommendations for future studies. Neurosci Biobehav Rev. (2019) 107:47-58. doi: 10.1016/j.neubiorev.2019.08.018

132. Berlim MT, van den Eynde F, Tovar-Perdomo S, Daskalakis ZJ. Response, remission and drop-out rates following high-frequency repetitive transcranial magnetic stimulation (rTMS) for treating major depression: a systematic review and meta-analysis of randomized, double-blind and sham-controlled trials. Psychol Med. (2014) 44:225-39. doi: 10.1017/S0033291713000512

133. Gaynes BN, Lloyd SW, Lux L, Gartlehner G, Hansen RA, Brode S, et al. Repetitive transcranial magnetic stimulation for treatment-resistant depression: a systematic review and meta-analysis. J Clin Psychiatry. (2014) 75:477-89; quiz 89. doi: 10.4088/JCP.13r08815

134. Slotema CW, Blom JD, Hoek HW, Sommer IE. Should we expand the toolbox of psychiatric treatment methods to include Repetitive Transcranial Magnetic Stimulation (rTMS)? A meta-analysis of the efficacy of rTMS in psychiatric disorders. J Clin Psychiatry. (2010) 71:87384. doi: 10.4088/JCP.08m04872gre

135. Senova S, Cotovio G, Pascual-Leone A, Oliveira-Maia AJ. Durability of antidepressant response to repetitive transcranial magnetic stimulation: systematic review and meta-analysis. Brain Stimul. (2019) 12:11928. doi: 10.1016/j.brs.2018.10.001

136. Rachid F. Maintenance repetitive transcranial magnetic stimulation (rTMS) for relapse prevention in with depression: a review. Psychiatry Res. (2018) 262:363-72. doi: 10.1016/j.psychres.2017.09.009

137. Chang J, Chu Y, Ren Y, Li C, Wang Y, Chu XP. Maintenance treatment of transcranial magnetic stimulation (TMS) for treatment-resistant depression 
patients responding to acute TMS treatment. Int J Physiol Pathophysiol Pharmacol. (2020) 12:128-33.

138. Hoogendam JM, Ramakers GM, Di Lazzaro V. Physiology of repetitive transcranial magnetic stimulation of the human brain. Brain Stimul. (2010) 3:95-118. doi: 10.1016/j.brs.2009. 10.005

139. Siebner HR, Rothwell J. Transcranial magnetic stimulation: new insights into representational cortical plasticity. Exp Brain Res. (2003) 148:116. doi: $10.1007 / \mathrm{s} 00221-002-1234-2$

140. Loo C, McFarquhar T, Walter G. Transcranial magnetic stimulation in adolescent depression. Australas Psychiatry. (2006) 14:81-5. doi: 10.1111/j.1440-1665.2006.02251.x

141. Walter G, Tormos JM, Israel JA, Pascual-Leone A. Transcranial magnetic stimulation in young persons: a review of known cases. J Child Adolesc Psychopharmacol. (2001) 11:69-75. doi: 10.1089/104454601750143483

142. Bloch Y, Grisaru N, Harel EV, Beitler G, Faivel N, Ratzoni G, et al. Repetitive transcranial magnetic stimulation in the treatment of depression in adolescents: an open-label study. J ECT. (2008) 24:1569. doi: 10.1097/YCT.0b013e318156aa49

143. Wall CA, Croarkin PE, Sim LA, Husain MM, Janicak PG, Kozel FA, et al. Adjunctive use of repetitive transcranial magnetic stimulation in depressed adolescents: a prospective, open pilot study. J Clin Psychiatry. (2011) 72:1263-9. doi: 10.4088/JCP.11m07003

144. Segev A, Spellun J, Bloch Y. Anxiety as a central outcome measure in an adolescent with major depressive disorder treated with repetitive transcranial magnetic stimulation. J ECT. (2014) 30:e54-5. doi: 10.1097/YCT.0000000000000183

145. Wall CA, Croarkin PE, Maroney-Smith MJ, Haugen LM, Baruth JM, Frye MA, et al. Magnetic resonance imaging-guided, open-label, highfrequency repetitive transcranial magnetic stimulation for adolescents with major depressive disorder. J Child Adolesc Psychopharmacol. (2016) 26:5829. doi: $10.1089 /$ cap.2015.0217

146. Wall CA, Croarkin PE, McClintock SM, Murphy LL, Bandel LA, Sim LA, et al. Neurocognitive effects of repetitive transcranial magnetic stimulation in adolescents with major depressive disorder. Front Psychiatry. (2013) 4:165. doi: 10.3389/fpsyt.2013.00165

147. Croarkin PE, Wall CA, Nakonezny PA, Buyukdura JS, Husain MM, Sampson $\mathrm{SM}$, et al. Increased cortical excitability with prefrontal high-frequency repetitive transcranial magnetic stimulation in adolescents with treatmentresistant major depressive disorder. J Child Adolesc Psychopharmacol. (2012) 22:56-64. doi: 10.1089/cap.2011.0054

148. Dhami P, Knyahnytska Y, Atluri S, Lee J, Courtney DB, Croarkin PE, et al. Feasibility and clinical effects of theta burst stimulation in youth with major depressive disorders: an open-label trial. J Affect Disord. (2019) 258:6673. doi: $10.1016 /$ j.jad.2019.07.084

149. MacMaster FP, Croarkin PE, Wilkes TC, McLellan Q, Langevin LM, Jaworska N, et al. Repetitive transcranial magnetic stimulation in youth with treatment resistant major depression. Front Psychiatry. (2019) 10:170. doi: 10.3389/fpsyt.2019.00170

150. Zhang T, Zhu J, Xu L, Tang X, Cui H, Wei Y, et al. Add-on rTMS for the acute treatment of depressive symptoms is probably more effective in adolescents than in adults: evidence from real-world clinical practice. Brain Stimul. (2019) 12:103-9. doi: 10.1016/j.brs.2018.09.007

151. Rosenich E, Gill S, Clarke P, Paterson T, Hahn L, Galletly C. Does rTMS reduce depressive symptoms in young people who have not responded to antidepressants? Early Interv Psychiatry. (2019) 13:112935. doi: 10.1111/eip.12743

152. Sonmez AI, Kucuker MU, Lewis CP, Kolla BP, Doruk Camsari D, Vande Voort JL, et al. Improvement in hypersomnia with high frequency repetitive transcranial magnetic stimulation in depressed adolescents: preliminary evidence from an open-label study. Prog Neuropsychopharmacol Biol Psychiatry. (2020) 97:109763. doi: 10.1016/j.pnpbp.2019.109763

153. Croarkin PE, Nakonezny PA, Deng ZD, Romanowicz M, Voort JLV, Camsari DD, et al. High-frequency repetitive TMS for suicidal ideation in adolescents with depression. J Affect Disord. (2018) 239:282-90. doi: 10.1016/j.jad.2018.06.048

154. Zhang T, Zhu J, Wang J, Tang Y, Xu L, Tang X, et al. An open-label trial of adjuvant high-frequency left prefrontal repetitive transcranial magnetic stimulation for treating suicidal ideation in adolescents and adults with depression. J ECT. (2020) doi: 10.1097/YCT.0000000000000739. [Epub ahead of print].

155. Zhang L, Zhu J, Zhang T, Jia Q, Hui L, Zhu H, et al. Comparative efficacy of add-on rTMS in treating the somatic and psychic anxiety symptoms of depression comorbid with anxiety in adolescents, adults, and elderly patients-A real-world clinical application. J Affect Disord. (2020) 276:30511. doi: 10.1016/j.jad.2020.05.151

156. Mayer G, Aviram S, Walter G, Levkovitz Y, Bloch Y. Long-term follow-up of adolescents with resistant depression treated with repetitive transcranial magnetic stimulation. J ECT. (2012) 28:84-6. doi: 10.1097/YCT.0b013e318238f01a

157. Sonmez AI, Camsari DD, Nandakumar AL, Voort JLV, Kung S, Lewis CP, et al. Accelerated TMS for depression: a systematic review and meta-analysis. Psychiatry Res. (2019) 273:770-81. doi: 10.1016/j.psychres.2018.12.041

158. Lakens D. Calculating and reporting effect sizes to facilitate cumulative science: a practical primer for t-tests and ANOVAs. Front Psychol. (2013) 4:863. doi: $10.3389 /$ fpsyg.2013.00863

159. Howard JP, Shun-Shin MJ, Hartley A, Bhatt DL, Krum H, Francis DP. Quantifying the 3 biases that lead to unintentional overestimation of the blood pressure-lowering effect of renal denervation. Circ Cardiovasc Qual Outcomes. (2016) 9:14-22. doi: 10.1161/CIRCOUTCOMES.115.002533

160. Davis NJ, Gold E, Pascual-Leone A, Bracewell RM. Challenges of proper placebo control for non-invasive brain stimulation in clinical and experimental applications. Eur J Neurosci. (2013) 38:2973-7. doi: 10.1111/ejn.12307

161. Hrobjartsson A, Gotzsche PC. Placebo interventions for all clinical conditions. Cochrane Database Syst Rev. (2010) 2010:CD003974. doi: 10.1002/14651858.CD003974.pub3

162. Razza LB, Moffa AH, Moreno ML, Carvalho AF, Padberg F, Fregni F, et al. A systematic review and meta-analysis on placebo response to repetitive transcranial magnetic stimulation for depression trials. Prog Neuropsychopharmacol Biol Psychiatry. (2018) 81:105-13. doi: 10.1016/j.pnpbp.2017.10.016

163. Brunoni AR, Lopes M, Kaptchuk TJ, Fregni F. Placebo response of non-pharmacological and pharmacological trials in major depression: a systematic review and meta-analysis. PLoS ONE. (2009) 4:e4824. doi: 10.1371/journal.pone.0004824

164. Lam RW, Chan P, Wilkins-Ho M, Yatham LN. Repetitive transcranial magnetic stimulation for treatment-resistant depression: a systematic review and metaanalysis. Can J Psychiatry. (2008) 53:621-31. doi: 10.1177/070674370805300909

165. Fergusson D, Glass KC, Waring D, Shapiro S. Turning a blind eye: the success of blinding reported in a random sample of randomised, placebo controlled trials. BMJ. (2004) 328:432. doi: 10.1136/bmj.328.74327.37952.631667.EE

166. Meister R, Abbas M, Antel J, Peters T, Pan Y, Bingel U, et al. Placebo response rates and potential modifiers in double-blind randomized controlled trials of second and newer generation antidepressants for major depressive disorder in children and adolescents: a systematic review and meta-regression analysis. Eur Child Adolesc Psychiatry. (2020) 29:25373. doi: 10.1007/s00787-018-1244-7

167. Keller MB, Ryan ND, Strober M, Klein RG, Kutcher SP, Birmaher B, et al. Efficacy of paroxetine in the treatment of adolescent major depression: a randomized, controlled trial. J Am Acad Child Adolesc Psychiatry. (2001) 40:762-72. doi: 10.1097/00004583-200107000-00010

168. Emslie GJ, Heiligenstein JH, Wagner KD, Hoog SL, Ernest DE, Brown E, et al. Fluoxetine for acute treatment of depression in children and adolescents: a placebo-controlled, randomized clinical trial. J Am Acad Child Adolesc Psychiatry. (2002) 41:1205-15. doi: 10.1097/00004583-200210000-00010

169. Wagner KD, Ambrosini P, Rynn M, Wohlberg C, Yang R, Greenbaum MS, et al. Efficacy of sertraline in the treatment of children and adolescents with major depressive disorder: two randomized controlled trials. JAMA. (2003) 290:1033-41. doi: 10.1001/jama.290.8.1033

170. March J, Silva S, Petrycki S, Curry J, Wells K, Fairbank J, et al. Fluoxetine, cognitive-behavioral therapy, and their combination for adolescents with depression: Treatment for Adolescents With Depression Study (TADS) randomized controlled trial. JAMA. (2004) 292:80720. doi: 10.1001/jama.292.7.807 
171. Berard R, Fong R, Carpenter DJ, Thomason C, Wilkinson C. An international, multicenter, placebo-controlled trial of paroxetine in adolescents with major depressive disorder. J Child Adolesc Psychopharmacol. (2006) 16:59-75. doi: 10.1089/cap.2006.16.59

172. von Knorring AL, Olsson GI, Thomsen PH, Lemming OM, Hulten A. A randomized, double-blind, placebo-controlled study of citalopram in adolescents with major depressive disorder. J Clin Psychopharmacol. (2006) 26:311-5. doi: 10.1097/01.jcp.0000219051.40632.d5

173. Usala T, Clavenna A, Zuddas A, Bonati M. Randomised controlled trials of selective serotonin reuptake inhibitors in treating depression in children and adolescents: a systematic review and meta-analysis. Eur Neuropsychopharmacol. (2008) 18:62-73. doi: 10.1016/j.euroneuro.2007.06.001

174. Bridge JA, Birmaher B, Iyengar S, Barbe RP, Brent DA. Placebo response in randomized controlled trials of antidepressants for pediatric major depressive disorder. Am J Psychiatry. (2009) 166:42-9. doi: 10.1176/appi.ajp.2008.08020247

175. Muthen B, Brown HC, Hunter AM, Cook IA, Leuchter AF. General approaches to analysis course: applying growth mixture modeling to randomized trials of depression medication. In: Shrout PE, editor. Causality and Psychopathology: Finding the Determinants of Disorders and their Cures. Washington, DC: American Psychiatric Publishing (2010). p. 15978. doi: 10.1093/oso/9780199754649.003.0012

176. Hunter AM, Muthen BO, Cook IA, Leuchter AF. Antidepressant response trajectories and quantitative electroencephalography (QEEG) biomarkers in major depressive disorder. J Psychiatr Res. (2010) 44:908. doi: 10.1016/j.jpsychires.2009.06.006

177. Brunoni AR, Chaimani A, Moffa AH, Razza LB, Gattaz WF, Daskalakis ZJ, et al. Repetitive transcranial magnetic stimulation for the acute treatment of major depressive episodes: a systematic review with network meta-analysis. JAMA Psychiatry. (2017) 74:143-52. doi: 10.1001/jamapsychiatry.2016.3644

178. Chung SW, Lewis BP, Rogasch NC, Saeki T, Thomson RH, Hoy KE, et al. Demonstration of short-term plasticity in the dorsolateral prefrontal cortex with theta burst stimulation: a TMS-EEG study. Clin Neurophysiol. (2017) 128:1117-26. doi: 10.1016/j.clinph.2017.04.005

179. Lee CW, Wu HF, Chu MC, Chung YJ, Mao WC, Li CT, et al. Mechanism of intermittent theta-burst stimulation in synaptic pathology in the prefrontal cortex in an antidepressant-resistant depression rat model. Cereb Cortex. (2021) 31:575-90. doi: 10.1093/cercor/bhaa244

180. Iwabuchi SJ, Raschke F, Auer DP, Liddle PF, Lankappa ST, Palaniyappan L. Targeted transcranial theta-burst stimulation alters fronto-insular network and prefrontal GABA. Neuroimage. (2017) 146:395-403. doi: 10.1016/j.neuroimage.2016.09.043

181. Viejo-Sobera R, Redolar-Ripoll D, Boixados M, Palaus M, ValeroCabre A, Marron EM. Impact of prefrontal theta burst stimulation on clinical neuropsychological tasks. Front Neurosci. (2017) 11:462. doi: $10.3389 /$ fnins.2017.00462

182. Blumberger DM, Vila-Rodriguez F, Thorpe KE, Feffer K, Noda Y, Giacobbe P, et al. Effectiveness of theta burst versus high-frequency repetitive transcranial magnetic stimulation in patients with depression (THREE-D): a randomised non-inferiority trial. Lancet. (2018) 391:168392. doi: 10.1016/S0140-6736(18)30295-2

183. Berlim MT, McGirr A, Rodrigues Dos Santos N, Tremblay S, Martins R. Efficacy of theta burst stimulation (TBS) for major depression: an exploratory meta-analysis of randomized and sham-controlled trials. J Psychiatr Res. (2017) 90:102-9. doi: 10.1016/j.jpsychires.2017.02.015

184. Herwig U, Padberg F, Unger J, Spitzer M, Schonfeldt-Lecuona C. Transcranial magnetic stimulation in therapy studies: examination of the reliability of "standard" coil positioning by neuronavigation. Biol Psychiatry. (2001) 50:58-61. doi: 10.1016/S0006-3223(01)01153-2

185. Sparing R, Buelte D, Meister IG, Paus T, Fink GR. Transcranial magnetic stimulation and the challenge of coil placement: a comparison of conventional and stereotaxic neuronavigational strategies. Hum Brain Mapp. (2008) 29:82-96. doi: 10.1002/hbm.20360

186. Nauczyciel C, Hellier P, Morandi X, Blestel S, Drapier D, Ferre JC, et al. Assessment of standard coil positioning in transcranial magnetic stimulation in depression. Psychiatry Res. (2011) 186:2328. doi: 10.1016/j.psychres.2010.06.012
187. Nikolin S, D'Souza O, Vulovic V, Alonzo A, Chand N, Dong V, et al. comparison of site localization techniques for brain stimulation. J ECT. (2019) 35:127-32. doi: 10.1097/YCT.0000000000000537

188. Trapp NT, Bruss J, King Johnson M, Uitermarkt BD, Garrett L, Heinzerling A, et al. Reliability of targeting methods in TMS for depression: Beam F3 vs. $5.5 \mathrm{~cm}$. Brain Stimul. (2020) 13:578-81. doi: 10.1016/j.brs.2020. 01.010

189. Kaiser RH, Whitfield-Gabrieli S, Dillon DG, Goer F, Beltzer M, Minkel J, et al. Dynamic resting-state functional connectivity in major depression. Neuropsychopharmacology. (2016) 41:1822-30. doi: 10.1038/npp.20 15.352

190. O'Shea J, Walsh V. Transcranial magnetic stimulation. Curr Biol. (2007) 17:R196-9. doi: 10.1016/j.cub.2007.01.030

191. Gordon EM, Laumann TO, Adeyemo B, Petersen SE. Individual variability of the system-level organization of the human brain. Cereb Cortex. (2017) 27:386-99. doi: 10.1093/cercor/bhv239

192. Sale MV, Mattingley JB, Zalesky A, Cocchi L. Imaging human brain networks to improve the clinical efficacy of non-invasive brain stimulation. Neurosci Biobehav Rev. (2015) 57:187-98. doi: 10.1016/j.neubiorev.2015.09.010

193. Teng S, Guo Z, Peng H, Xing G, Chen H, He B, et al. High-frequency repetitive transcranial magnetic stimulation over the left DLPFC for major depression: session-dependent efficacy: a meta-analysis. Eur Psychiatry. (2017) 41:75-84. doi: 10.1016/j.eurpsy.2016.11.002

194. Fitzgerald PB, Hoy KE, Elliot D, McQueen S, Wambeek LE, Daskalakis ZJ. Exploring alternative rTMS strategies in non-responders to standard high frequency left-sided treatment: a switching study. J Affect Disord. (2018) 232:79-82. doi: 10.1016/j.jad.2018.02.016

195. Schulze L, Feffer K, Lozano C, Giacobbe P, Daskalakis ZJ, Blumberger $\mathrm{DM}$, et al. Number of pulses or number of sessions? An open-label study of trajectories of improvement for once-vs. twice-daily dorsomedial prefrontal rTMS in major depression. Brain Stimul. (2018) 11:32736. doi: 10.1016/j.brs.2017.11.002

196. Fitzgerald PB, Hoy KE, Elliot D, Susan McQueen RN, Wambeek LE, Daskalakis ZJ. Accelerated repetitive transcranial magnetic stimulation in the treatment of depression. Neuropsychopharmacology. (2018) 43:156572. doi: 10.1038/s41386-018-0009-9

197. Chen L, Hudaib AR, Hoy KE, Fitzgerald PB. Efficacy, efficiency and safety of high-frequency repetitive transcranial magnetic stimulation applied more than once a day in depression: a systematic review. J Affect Disord. (2020) 277:986-96. doi: 10.1016/j.jad.2020.09.035

198. Cole EJ, Stimpson KH, Bentzley BS, Gulser M, Cherian K, Tischler C, et al. Stanford accelerated intelligent neuromodulation therapy for treatment-resistant depression. Am J Psychiatry. (2020) 177:716-26. doi: 10.1176/appi.ajp.2019.19070720

199. Rachid F. Accelerated transcranial magnetic stimulation for the treatment of patients with depression: a review. Asian J Psychiatr. (2019) 40:715. doi: 10.1016/j.ajp.2019.02.003

200. Baeken C, Marinazzo D, Wu GR, Van Schuerbeek P, De Mey J, Marchetti I, et al. Accelerated HF-rTMS in treatment-resistant unipolar depression: insights from subgenual anterior cingulate functional connectivity. World J Biol Psychiatry. (2014) 15:286-97. doi: 10.3109/15622975.2013. 872295

201. Lynch G, Kramar EA, Babayan AH, Rumbaugh G, Gall CM. Differences between synaptic plasticity thresholds result in new timing rules for maximizing long-term potentiation. Neuropharmacology. (2013) 64:2736. doi: 10.1016/j.neuropharm.2012.07.006

202. Thomson AC, Sack AT. How to design optimal accelerated rTMS protocols capable of promoting therapeutically beneficial metaplasticity. Front Neurol. (2020) 11:599918. doi: 10.3389/fneur.2020.5 99918

203. Moreno C, Wykes T, Galderisi S, Nordentoft M, Crossley N, Jones $\mathrm{N}$, et al. How mental health care should change as a consequence of the COVID-19 pandemic. Lancet Psychiatry. (2020) 7:813-24. doi: 10.1016/S2215-0366(20)30307-2

204. Breslau J, Gilman SE, Stein BD, Ruder T, Gmelin T, Miller E. Sex differences in recent first-onset depression in an epidemiological sample of adolescents. Transl Psychiatry. (2017) 7:e1139. doi: 10.1038/tp. 2017.105 
205. Chen J, Yu J, Zhang L, Li X, Zhang J. Etiological heterogeneity of symptom dimensions of adolescent depression. Psych J. (2014) 3:25463. doi: $10.1002 /$ pchj.62

206. Yaroslavsky I, Pettit JW, Lewinsohn PM, Seeley JR, Roberts RE. Heterogeneous trajectories of depressive symptoms: adolescent predictors and adult outcomes. J Affect Disord. (2013) 148:3919. doi: 10.1016/j.jad.2012.06.028

207. Mojtabai R, Olfson M, Han B. National trends in the prevalence and treatment of depression in adolescents and young adults. Pediatrics. (2016) 138:e20161878. doi: 10.1542/peds.2016-1878

208. Stringaris A. Editorial: what is depression? J Child Psychol Psychiatry. (2017) 58:1287-9. doi: 10.1111/jcpp.12844

209. Nielson DM, Keren H, O'Callaghan G, Jackson SM, Douka I, Vidal-Ribas P, et al. Great expectations: a critical review of and suggestions for the study of reward processing as a cause and predictor of depression. Biol Psychiatry. (2020) 89:134-43 doi: 10.1101/2020.03.04.975136

210. Bozzay ML, Primack J, Barredo J, Philip NS. Transcranial magnetic stimulation to reduce suicidality - a review and naturalistic outcomes. $J$ Psychiatr Res. (2020) 125:106-12. doi: 10.1016/j.jpsychires.2020.03.016

211. Dinga R, Schmaal L, Penninx B, van Tol MJ, Veltman DJ, van Velzen L, et al. Evaluating the evidence for biotypes of depression: methodological replication and extension of. Neuroimage Clin. (2019) 22:101796. doi: 10.1016/j.nicl.2019.101796

212. Henderson SE, Johnson AR, Vallejo AI, Katz L, Wong E, Gabbay V. A preliminary study of white matter in adolescent depression: relationships with illness severity, anhedonia, and irritability. Front Psychiatry. (2013) 4:152. doi: $10.3389 /$ fpsyt.2013.00152

213. Rzepa E, McCabe C. Anhedonia and depression severity dissociated by dmPFC resting-state functional connectivity in adolescents. J Psychopharmacol. (2018) 32:1067-74. doi: 10.1177/0269881118799935

214. Stringaris A, Vidal-Ribas Belil P, Artiges E, Lemaitre H, Gollier-Briant F, Wolke S, et al. The Brain's response to reward anticipation and depression in adolescence: dimensionality, specificity, and longitudinal predictions in a community-based sample. Am J Psychiatry. (2015) 172:121523. doi: 10.1176/appi.ajp.2015.14101298

215. Pornpattananangkul N, Leibenluft E, Pine DS, Stringaris A. association between childhood anhedonia and alterations in large-scale resting-state networks and task-evoked activation. JAMA Psychiatry. (2019) 76:62433. doi: $10.1001 /$ jamapsychiatry.2019.0020

216. Krystal AD, Pizzagalli DA, Smoski M, Mathew SJ, Nurnberger J Jr, Lisanby $\mathrm{SH}$, et al. A randomized proof-of-mechanism trial applying the 'fastfail' approach to evaluating kappa-opioid antagonism as a treatment for anhedonia. Nat Med. (2020) 26:760-8. doi: 10.1038/s41591-020-0806-7

217. Abdelnaim MA, Langguth B, Deppe M, Mohonko A, Kreuzer PM, Poeppl TB, et al. Anti-suicidal efficacy of repetitive transcranial magnetic stimulation in depressive patients: a retrospective analysis of a large sample. Front Psychiatry. (2019) 10:929. doi: 10.3389/fpsyt.2019.00929

218. Philip NS, Dunner DL, Dowd SM, Aaronson ST, Brock DG, Carpenter LL, et al. Can medication free, treatment-resistant, depressed patients who initially respond to TMS Be maintained off medications? A prospective, 12-month multisite randomized pilot study. Brain Stimul. (2016) 9:2517. doi: 10.1016/j.brs.2015.11.007

219. Best SRD, Pavel DG, Haustrup N. Combination therapy with transcranial magnetic stimulation and ketamine for treatment-resistant depression: a long-term retrospective review of clinical use. Heliyon. (2019) 5:e2187. doi: 10.1016/j.heliyon.2019.e02187

220. Romei V, Brodbeck V, Michel C, Amedi A, Pascual-Leone A, Thut G. Spontaneous fluctuations in posterior alpha-band EEG activity reflect variability in excitability of human visual areas. Cereb Cortex. (2008) 18:2010-8. doi: 10.1093/cercor/bhm229

221. Facchini S, Muellbacher W, Battaglia F, Boroojerdi B, Hallett M. Focal enhancement of motor cortex excitability during motor imagery: a transcranial magnetic stimulation study. Acta Neurol Scand. (2002) 105:14651. doi: $10.1034 /$ j.1600-0404.2002.10004.x

222. Li S, Latash ML, Zatsiorsky VM. Effects of motor imagery on finger force responses to transcranial magnetic stimulation. Brain Res Cogn Brain Res. (2004) 20:273-80. doi: 10.1016/j.cogbrainres.2004. 03.003
223. Scangos KW, Makhoul GS, Sugrue LP, Chang EF, Krystal AD. Statedependent responses to intracranial brain stimulation in a patient with depression. Nat Med. (2021) 27:229-31. doi: 10.1038/s41591-020-01175-8

224. Tendler A, Sisko E, Barnea-Ygael N, Zangen A, Storch EA. A method to provoke obsessive compulsive symptoms for basic research and clinical interventions. Front Psychiatry. (2019) 10:814. doi: 10.3389/fpsyt.2019.00814

225. Isserles M, Shalev AY, Roth Y, Peri T, Kutz I, Zlotnick E, et al. Effectiveness of deep transcranial magnetic stimulation combined with a brief exposure procedure in post-traumatic stress disorder-a pilot study. Brain Stimul. (2013) 6:377-83. doi: 10.1016/j.brs.2012.07.008

226. Dinur-Klein L, Dannon P, Hadar A, Rosenberg O, Roth Y, Kotler $\mathrm{M}$, et al. Smoking cessation induced by deep repetitive transcranial magnetic stimulation of the prefrontal and insular cortices: a prospective, randomized controlled trial. Biol Psychiatry. (2014) 76:742-9. doi: 10.1016/j.biopsych.2014.05.020

227. Carmi L, Tendler A, Bystritsky A, Hollander E, Blumberger DM, Daskalakis J, et al. Efficacy and safety of deep transcranial magnetic stimulation for obsessive-compulsive disorder: a prospective multicenter randomized double-blind placebo-controlled trial. Am J Psychiatry. (2019) 176:9318. doi: 10.1176/appi.ajp.2019.18101180

228. Sathappan AV, Luber BM, Lisanby SH. The dynamic duo: combining noninvasive brain stimulation with cognitive interventions. Prog Neuropsychopharmacol Biol Psychiatry. (2019) 89:347-60. doi: 10.1016/j.pnpbp.2018.10.006

229. Feffer K, Lee HH, Mansouri F, Giacobbe P, Vila-Rodriguez F, Kennedy $\mathrm{SH}$, et al. Early symptom improvement at 10 sessions as a predictor of rTMS treatment outcome in major depression. Brain Stimul. (2018) 11:1819. doi: 10.1016/j.brs.2017.10.010

230. Harris AD, Saleh MG, Edden RA. Edited (1) H magnetic resonance spectroscopy in vivo: Methods and metabolites. Magn Reson Med. (2017) 77:1377-89. doi: 10.1002/mrm.26619

231. Saleh MG, Oeltzschner G, Chan KL, Puts NAJ, Mikkelsen M, Schar M, et al. Simultaneous edited MRS of GABA and glutathione. Neuroimage. (2016) 142:576-82. doi: 10.1016/j.neuroimage.2016.07.056

232. Jalbrzikowski M, Liu F, Foran W, Roeder K, Devlin B, Luna B. Resting-state functional network organization is stable across adolescent development for typical and psychosis spectrum youth. Schizophr Bull. (2020) 46:395407. doi: $10.1093 / \mathrm{schbul} / \mathrm{sbz} 053$

233. Jalbrzikowski M, Liu F, Foran W, Klei L, Calabro FJ, Roeder K, et al. Functional connectome fingerprinting accuracy in youths and adults is similar when examined on the same day and 1.5-years apart. Hum Brain Mapp. (2020) 41:4187-99. doi: 10.1101/812719

234. Marek S, Tervo-Clemmens B, Nielsen AN, Wheelock MD, Miller RL, Laumann $\mathrm{TO}$, et al. Identifying reproducible individual differences in childhood functional brain networks: an ABCD study. Dev Cogn Neurosci. (2019) 40:100706. doi: 10.1016/j.dcn.2019.100706

235. Ho TC, Connolly CG, Henje Blom E, LeWinn KZ, Strigo IA, Paulus MP, et al. Emotion-dependent functional connectivity of the default mode network in adolescent depression. Biol Psychiatry. (2015) 78:63546. doi: 10.1016/j.biopsych.2014.09.002

236. Kuhn S, Vanderhasselt MA, De Raedt R, Gallinat J. Why ruminators won't stop: the structural and resting state correlates of rumination and its relation to depression. J Affect Disord. (2012) 141:352-60. doi: 10.1016/j.jad.2012.03.024

237. Cullen KR, Westlund MK, Klimes-Dougan B, Mueller BA, Houri A, Eberly LE, et al. Abnormal amygdala resting-state functional connectivity in adolescent depression. JAMA Psychiatry. (2014) 71:1138-47. doi: 10.1001/jamapsychiatry.2014.1087

238. Clark DL, Konduru N, Kemp A, Bray S, Brown EC, Goodyear $\mathrm{B}$, et al. The impact of age of onset on amygdala intrinsic connectivity in major depression. Neuropsychiatr Dis Treat. (2018) 14:343-52. doi: 10.2147/NDT.S145042

239. Connolly CG, Ho TC, Blom EH, LeWinn KZ, Sacchet MD, Tymofiyeva O, et al. Resting-state functional connectivity of the amygdala and longitudinal changes in depression severity in adolescent depression. J Affect Disord. (2017) 207:86-94. doi: 10.1016/j.jad.2016.09.026

240. Scheuer H, Alarcon G, Demeter DV, Earl E, Fair DA, Nagel BJ. Reduced fronto-amygdalar connectivity in adolescence is associated with increased 
depression symptoms over time. Psychiatry Res Neuroimaging. (2017) 266:35-41. doi: 10.1016/j.pscychresns.2017.05.012

241. Cui Z, Li H, Xia CH, Larsen B, Adebimpe A, Baum GL, et al. Individual variation in functional topography of association networks in youth. Neuron. (2020) 106:340-53 e8. doi: 10.1016/j.neuron.2020.01.029

242. Noble S, Scheinost D, Constable RT. A decade of test-retest reliability of functional connectivity: A systematic review and meta-analysis. Neuroimage. (2019) 203:116157. doi: 10.1016/j.neuroimage.2019.116157

243. Gratton C, Laumann TO, Nielsen AN, Greene DJ, Gordon EM, Gilmore $\mathrm{AW}$, et al. Functional brain networks are dominated by stable group and individual factors, not cognitive or daily variation. Neuron. (2018) 98:439-52 e5. doi: 10.1016/j.neuron.2018.03.035

244. Birn RM, Molloy EK, Patriat R, Parker T, Meier TB, Kirk GR, et al. The effect of scan length on the reliability of resting-state fMRI connectivity estimates. Neuroimage. (2013) 83:550-8. doi: 10.1016/j.neuroimage.2013. 05.099

245. Avissar M, Powell F, Ilieva I, Respino M, Gunning FM, Liston C, et al. Functional connectivity of the left DLPFC to striatum predicts treatment response of depression to TMS. Brain Stimul. (2017) 10:91925. doi: 10.1016/j.brs.2017.07.002

246. Baeken C, Duprat R, Wu GR, De Raedt R, van Heeringen K. Subgenual anterior cingulate-medial orbitofrontal functional connectivity in medication-resistant major depression: a neurobiological marker for accelerated intermittent theta burst stimulation treatment? Biol Psychiatry Cogn Neurosci Neuroimaging. (2017) 2:556-65. doi: 10.1016/j.bpsc.2017.01.001

247. Cash RFH, Cocchi L, Anderson R, Rogachov A, Kucyi A, Barnett AJ, et al. A multivariate neuroimaging biomarker of individual outcome to transcranial magnetic stimulation in depression. Hum Brain Mapp. (2019) 40:4618-29. doi: 10.1002/hbm.24725

248. Cash RFH, Zalesky A, Thomson RH, Tian Y, Cocchi L, Fitzgerald PB. Subgenual functional connectivity predicts antidepressant treatment response to transcranial magnetic stimulation: independent validation and evaluation of personalization. Biol Psychiatry. (2019) 86:e5-e7. doi: 10.1016/j.biopsych.2018.12.002

249. Ge R, Blumberger DM, Downar J, Daskalakis ZJ, Dipinto AA, Tham JCW, et al. Abnormal functional connectivity within resting-state networks is related to rTMS-based therapy effects of treatment resistant depression: a pilot study. J Affect Disord. (2017) 218:75-81. doi: 10.1016/j.jad.2017.04.060

250. Ge R, Downar J, Blumberger DM, Daskalakis ZJ, Vila-Rodriguez F. Functional connectivity of the anterior cingulate cortex predicts treatment outcome for rTMS in treatment-resistant depression at 3-month follow-up. Brain Stimul. (2020) 13:206-14. doi: 10.1016/j.brs.2019.10.012

251. Klooster DC, Vos IN, Caeyenberghs K, Leemans A, David S, Besseling $\mathrm{RM}$, et al. Indirect frontocingulate structural connectivity predicts clinical response to accelerated rTMS in major depressive disorder. J Psychiatry Neurosci. (2020) 45:243-52. doi: 10.1503/jpn.190088
252. Salomons TV, Dunlop K, Kennedy SH, Flint A, Geraci J, Giacobbe $\mathrm{P}$, et al. Resting-state cortico-thalamic-striatal connectivity predicts response to dorsomedial prefrontal rTMS in major depressive disorder. Neuropsychopharmacology. (2014) 39:488-98. doi: 10.1038/npp.2013.222

253. Weigand A, Horn A, Caballero R, Cooke D, Stern AP, Taylor SF, et al. Prospective validation that subgenual connectivity predicts antidepressant efficacy of transcranial magnetic stimulation sites. Biol Psychiatry. (2018) 84:28-37. doi: 10.1016/j.biopsych.2017.10.028

254. Eshel N, Keller CJ, Wu W, Jiang J, Mills-Finnerty C, Huemer J, et al. Global connectivity and local excitability changes underlie antidepressant effects of repetitive transcranial magnetic stimulation. Neuropsychopharmacology. (2020) 45:1018-25. doi: 10.1038/s41386-020-0633-z

255. Lener MS, Iosifescu DV. In pursuit of neuroimaging biomarkers to guide treatment selection in major depressive disorder: a review of the literature. Ann N Y Acad Sci. (2015) 1344:50-65. doi: 10.1111/nyas.12759

256. Pizzagalli DA. Frontocingulate dysfunction in depression: toward biomarkers of treatment response. Neuropsychopharmacology. (2011) 36:183-206. doi: 10.1038/npp.2010.166

257. Brakowski J, Spinelli S, Dorig N, Bosch OG, Manoliu A, Holtforth MG, et al. Resting state brain network function in major depression - Depression symptomatology, antidepressant treatment effects, future research. $J$ Psychiatr Res. (2017) 92:147-59. doi: 10.1016/j.jpsychires.2017.04.007

258. Klimes-Dougan B, Westlund Schreiner M, Thai M, Gunlicks-Stoessel M, Reigstad K, Cullen KR. Neural and neuroendocrine predictors of pharmacological treatment response in adolescents with depression: a preliminary study. Prog Neuropsychopharmacol Biol Psychiatry. (2018) 81:194-202. doi: 10.1016/j.pnpbp.2017.10.015

259. Grabb MC, Hillefors M, Potter WZ. The NIMH 'Fast-Fail Trials' (FAST) initiative: rationale, promise, and progress. Pharmaceut Med. (2020) 34:23345. doi: 10.1007/s40290-020-00343-y

Disclaimer: The views expressed in this article do not necessarily represent the views of the National Institutes of Health, the Department of Health and Human Services, or the United States Government. LO is a paid scientific advisor to Achieve TMS and Anavex Life Sciences.

Conflict of Interest: The authors declare that the research was conducted in the absence of any commercial or financial relationships that could be construed as a potential conflict of interest.

Copyright $\odot 2021$ Oberman, Hynd, Nielson, Towbin, Lisanby and Stringaris. This is an open-access article distributed under the terms of the Creative Commons Attribution License (CC BY). The use, distribution or reproduction in other forums is permitted, provided the original author(s) and the copyright owner(s) are credited and that the original publication in this journal is cited, in accordance with accepted academic practice. No use, distribution or reproduction is permitted which does not comply with these terms. 